\title{
INVARIANT MEASURES AND EQUILIBRIUM STATES FOR SOME MAPPINGS WHICH EXPAND DISTANCES

\author{
BY
}

PETER WALTERS

\begin{abstract}
Aestract. For a certain collection of transformations $T$ we define a Perron-Frobenius operator and prove a convergence theorem for the powers of the operator along the lines of the theorem D. Ruelle proved in his investigation of the equilibrium states of one-dimensional lattice systems. We use the convergence theorem to study the existence and ergodic properties of equilibrium states for $T$ and also to study the problem of invariant measures for $T$. Examples of the transformations $T$ considered are expanding maps, transformations arising from $f$-expansions and shift systems.
\end{abstract}

We study the problems of invariant measures and existence of unique equlibrium states for a certain class of transformations which includes expanding maps, subshifts of finite type, shifts on an infinite alphabet, and the transformations arising from $f$-expansions. The results about invariant measures and equilibrium states are deduced from a generalized PerronFrobenius theorem (Theorem 8) of the type proved by D. Ruelle [20]. R. Sacksteder proved related convergence theorems for the case of $f$-expansions [21] and the case of expanding maps [22] but neither of his results cover both cases. The invariant measures and equilibrium states we obtain can be characterized by a variational principle and in all the above examples have Bernoulli natural extensions. For example if $T$ is a $C^{2}$ expanding map on a compact connected manifold then $T$ preserves a probability measure $\mu$ equivalent to the smooth measures and the natural extension of $(T, \mu)$ is a Bernoulli shift (Theorem 18). The existence of $\mu$ was also shown in [11] and [22]. Any real-valued Hölder continuous function $\varphi$ has a unique equilibrium state $\mu_{\varphi}$ for an expanding map $T$ and the natural extension of $\left(T, \mu_{\varphi}\right)$ is a Bernoulli shift (Theorem 19). We do not have to use a symbolic representation to get the measure $\mu$.

When $T$ is a transformation arising from an $f$-expansion we show that the invariant measure equivalent to Lebesgue measure has a Bernoulli natural extension and is given by a variational principle (Theorem 22). The Bernoulli

Received by the editors March 25, 1976.

AMS (MOS) subject classifications (1970). Primary 28A65; Secondary 58F15, 82A25.

$K e y$ words and phrases. Perron-Frobenius operator, equilibrium state, Bernoulli shift, expanding map, f-expansion.

- American Mathematical Society 1978 
property was proved in [19] and [2]. Also certain functions have unique equilibrium states for $T$. Our results could also be used to give some of the results on multidimensional $f$-expansions [27].

Our convergence theorem (Theorem 8) generalizes the Ruelle-PerronFrobenius theorem ([3], [5], [24]) and so implies the results on equilibrium states for subshifts of finite type. It also gives results for the case of shifts on infinite alphabets (\$3.3). Using these results we give an example (which appears in [6] and [9]) of a continuous function with exactly two ergodic equilibrium states for the shift transformation on the product space $\Pi_{0}^{\infty}\{0,1\}$. We also deduce a Perron-Frobenius theorem for infinite matrices (Theorem 25).

We now summarize the notation used. We shall have a compact metric space $\bar{X}$ and an open dense subset $X$ of it. $C(\bar{X})$ will denote the Banach space of real-valued continuous functions on $\bar{X}$ with the supremum norm. $\|f\|$ will denote the norm of $f$, and " $\rightarrow$ " will denote convergence in the supremum norm. $C(X)$ will denote the space of continuous functions on $X$ and $U C(X)$ will denote the space of uniformly continuous functions on $X . M(\bar{X})$ will denote the collection of all probability measures on the $\sigma$-algebra of Borel sets $\mathscr{B}$ of $\bar{X}$. Since $\bar{X}$ is a compact metric space, $M(\bar{X})$ is a compact convex metrisable space in the weak* topology. We shall use $\bar{E}$ to denote the closure of a set $E$, and $B_{\varepsilon}(x)$ will denote the ball centered at $x$ of radius $\varepsilon$. $\partial E$ will denote the boundary of $E$ (i.e., $\partial E=\bar{E} \backslash \operatorname{int}(E)$ ). $\operatorname{diam}(E)$ will denote the diameter of $E$.

If $\mu \in M(\bar{X})$ and $f \in C(\bar{X})$ we shall let $\mu(f)$ denote the integral of $f$ with respect to $\mu$. If $h \in C(\bar{X}), h \cdot \mu$ will denote the measure defined by $(h \cdot \mu)(f)$ $=\mu(h \cdot f), f \in C(\bar{X})$. We shall use the notation for entropy from [18] or [26]. If $T$ is a transformation preserving the measure $\mu$ then $H_{\mu}(\xi), h_{\mu}(T, \xi), h_{\mu}(T)$, $H_{\mu}(\eta / \xi)$ will denote the entropy functions as defined in [18] or [26].

If $X_{0} \subset X, T: X_{0} \rightarrow X$ is a map and $\varphi: X \rightarrow R$ is a map we write $S_{n} \varphi(x)$ for $\sum_{i=0}^{n-1} \varphi\left(T^{i} x\right)$. A continuous map $T: \bar{X} \rightarrow \bar{X}$ of a compact space is called expansive (sometimes one-sided expansive) if there exists $\delta>0$ with the property that $d\left(T^{n} x, T^{n} y\right)<\delta_{0} \forall n>0$ implies $x=y$. An equivalent definition is to require the existence of an open cover $\alpha$ of $\bar{X}$ with the property that $\bigcap_{n=0}^{\infty} T^{-n} \bar{A}_{i_{n}}$ contains at most one point for all choices of $\left\{A_{i_{n}}\right\}$ from $\alpha$ [26].

1. Convergence theorems. Let $\bar{X}$ be a compact metric space with metric $d$ and let $X$ be an open dense subset of $\bar{X}$. Let $X_{0}$ be an open dense subset of $X$ and suppose $T: X_{0} \rightarrow X$ is a continuous map of $X_{0}$ onto $X$ such that the set $\left\{T^{-1} x\right\}$ is at most countable for each $x \in X$. In some applications all three spaces $X_{0}, X, \bar{X}$ coincide. We shall always assume $T$ has the following property: 
I. There exists $\varepsilon_{0}>0$ such that for every $x \in X, T^{-1}\left(B_{2 \varepsilon_{0}}(x) \cap X\right)$ can be written uniquely as a disjoint union of a finite or countable number of open subsets $A_{1}(x), A_{2}(x), \ldots$ of $X_{0}$ with for each $i,\left.T\right|_{A_{1}(x)}$ being a homeomorphism of $A_{i}(x)$ onto $B_{2 \varepsilon_{0}}(x) \cap X$ not decreasing any distances (i.e., if $y$, $\left.y^{\prime} \in A_{i}(x), d\left(T y, T y^{\prime}\right)>d\left(y, y^{\prime}\right)\right)$. We shall call the sets $A_{i}(x)$ the components of $T^{-1}\left(B_{2 \varepsilon_{0}}(x) \cap X\right)$. Note that if $d\left(x, x^{\prime}\right)<\varepsilon_{0}$ there is a natural bijection between the sets $\left\{T^{-1} x\right\}$ and $\left\{T^{-1} x^{\prime}\right\}$ by letting $y \in T^{-1} x$ correspond to $y^{\prime} \in T^{-1} x^{\prime}$ if $y$ and $y^{\prime}$ lie in the same $A_{i}(x)$. We shall use the notation $y^{\prime}$ to denote the point of $T^{-1} x^{\prime}$ corresponding to $y \in T^{-1} x$ when $d\left(x, x^{\prime}\right)<\varepsilon_{0}$. Notice that $d\left(y, y^{\prime}\right)<d\left(x, x^{\prime}\right)<\varepsilon_{0}$ so the elements of $\left\{T^{-1} y\right\}$ and $\left\{T^{-1} y^{\prime}\right\}$ are linked by a natural bijection too. This gives rise to a natural bijection between $\left\{T^{-2} x\right\}$ and $\left\{T^{-2} x^{\prime}\right\}$ and in the same way we arrive at a natural bijection between the sets $\left\{T^{-n} x\right\}$ and $\left\{T^{-n} x^{\prime}\right\}(n>1)$ when $d\left(x, x^{\prime}\right)<\varepsilon_{0}$. We use the notation $y^{\prime}$ for the point in $T^{-n} x^{\prime}$ corresponding to $y \in T^{-n} x$. (So for $j<n-1 \exists i$ such that $T^{j} y, T^{j} y^{\prime} \in A_{i j}\left(T^{j+1} y\right)$.)

We want to define a Ruelle-Perron-Frobenius operator (or transfer matrix operator) $\overline{\mathrm{L}}_{\varphi}: C(\bar{X}) \rightarrow C(\bar{X})$ for certain $\varphi \in C\left(X_{0}\right)$ by the formula $\overline{\mathcal{L}}_{\varphi} f(x)=$ $\Sigma_{y \in T^{-1} x} e^{\varphi(y)} f(y)$ if $x \in X$. We now consider conditions on $\varphi$ which allow us to do this.

Consider the following conditions on $\varphi \in C\left(X_{0}\right)$ :

(i) $\exists K$ such that $\Sigma_{y \in T^{-1} x} e^{\varphi(y)}<K \forall x \in X$,

(ii) $\Sigma_{y \in T^{-1} x}\left|e^{\varphi(y)}-e^{\varphi\left(y^{\prime}\right)}\right| \rightarrow 0$ as $d\left(x, x^{\prime}\right) \rightarrow 0$.

(Here the sum is over the finite or countable set $T^{-1} x$ and $y^{\prime}$ is the point of $T^{-1} x^{\prime}$ corresponding to $y \in T^{-1} x$.)

If $X_{0}=X=\bar{X}$ and $\left|T^{-1} x\right|<k \forall x \in \bar{X}$ then (i) and (ii) always hold for all $\varphi \in C(\bar{X})$.

If (ii) holds and we know $\Sigma_{y \in T^{-1} x} e^{\varphi(y)}<\infty$ for some $x$ then (i) holds.

LEMMA 1. Let $T: X_{0} \rightarrow X$ satisfy I and let $\varphi \in C\left(X_{0}\right)$ satisfy (i) and (ii). Then $\mathfrak{E}_{\varphi} f(x)=\Sigma_{y \in T^{-1} x} e^{\varphi(y)} f(y)$ defines a map $\mathfrak{E}_{\varphi}: U C(X) \rightarrow U C(X)$ which extends to a positive continuous linear map $\overline{\mathrm{L}}_{\varphi}: C(\bar{X}) \rightarrow C(\bar{X})$.

Proof. The sum in the definition of $\mathfrak{L}_{\varphi}$ converges by (i). If $f \in U C(X)$ then $\mathfrak{L}_{\varphi} f \in U C(X)$ since if $d\left(x, x^{\prime}\right)<\varepsilon_{0}$

$$
\begin{aligned}
\left|\mathfrak{L}_{\varphi} f(x)-\mathfrak{L}_{\varphi} f\left(x^{\prime}\right)\right|< & \|f\| \sum_{y \in T^{-1} x}\left|e^{\varphi(y)}-e^{\varphi\left(y^{\prime}\right)}\right| \\
& +K \sup _{y \in T^{-1} x_{x}}\left|f(y)-f\left(y^{\prime}\right)\right| .
\end{aligned}
$$

Let $\overline{\mathfrak{L}}_{\varphi}^{*}$ be the dual of $\overline{\mathfrak{e}}_{\varphi}$. The following will be useful in the study of equilibrium states.

Lemma 2. Let $T: X_{0} \rightarrow X$ satisfy I and let $\varphi \in C\left(X_{0}\right)$ satisfy (i) and (ii). 
There exists a real number $\lambda>0$ and $\nu \in M(\bar{X})$ such that $\overline{\mathcal{E}}_{\phi}^{*} \nu=\lambda \nu$.

Proof. The map $\mu \rightarrow \bar{L}_{\varphi}^{*} \mu /\left(\overline{\mathrm{L}}_{\varphi}^{*} \mu\right)(1)$ is a continuous map of $M(\bar{X})$ into $M(\bar{X})$ and hence has a fixed point $\nu$ by the Schauder-Tychonoff fixed point theorem. Let $\lambda=\left(\overline{\mathscr{L}}_{\varphi}^{*} \nu\right)(1)$.

LemMA 3. Let $\lambda>0$ and $\nu \in M(\bar{X})$ be as in Lemma 2. Let $A_{i}$ be $a$ component of $T^{-1}\left(B_{2 \varepsilon_{0}}(x) \cap X\right)$ and let $\left.\nu\right|_{A_{i}}$ be the measure $\nu$ restricted to $A_{i}$ $\left(\left(\left.\nu\right|_{A_{i}}\right)(E)=\nu\left(E \cap A_{i}\right)\right)$. Then the Radon-Nikody'm derivative

$$
\frac{\left.d(\nu T)\right|_{A_{i}}}{\left.d \nu\right|_{A_{i}}}(y)=\lambda e^{-\varphi(y)}
$$

for almost all $y \in A_{i}$.

Proof. Let $f \in C(\bar{X})$ have compact support inside $A_{i}$. Then

$$
\begin{aligned}
\lambda \int_{A_{i}} f d \nu & =\lambda \int f d \nu=\int \overline{\mathcal{L}}_{\varphi} f d \nu=\int_{T A_{i}} e^{\varphi\left(y_{i}\right)} f\left(y_{i}\right) d \nu(x) \\
& =\int_{A_{i}} e^{\varphi(x)} f(x) \frac{\left.d \nu T\right|_{A_{i}}}{\left.d \nu\right|_{A_{i}}}(x) d \nu(x)
\end{aligned}
$$

where $y_{i}$ is the element of $T^{-1} x$ inside $A_{i}$. The result follows.

Corollary 4. Let $\lambda>0$ and $\nu \in M(\bar{X})$ be as in Lemma 2. Then $\nu$ is positively nonsingular and nonsingular for T. (I.e., if $E \subset X_{0}$ and $\nu(E)=0$ then $\nu(T E)=0$; and if $F \subset X$ and $\nu(F)=0$ then $\nu\left(T^{-1} F\right)=0$.)

Proof. Since $\bar{X}$ is compact it is covered by a finite number of balls of radius $2 \varepsilon_{0}$, say $B_{2 \varepsilon_{0}}\left(x_{1}\right), \ldots, B_{2 \varepsilon_{0}}\left(x_{p}\right)$. Therefore $X$ is covered by the sets $B_{2 e_{0}}\left(x_{j}\right) \cap X, 1<j<p$, and $X_{0}$ is covered by the components of $T^{-1}\left(B_{2 \varepsilon_{0}}\left(x_{j}\right) \cap X\right), 1<j<p$. To show $T$ is positively nonsingular it suffices to show $\nu(E)=0$ implies $\nu(T E)=0$ when $E$ is a subset of some component $A_{i}$ of some $T^{-1}\left(B_{2 \varepsilon_{0}}\left(x_{j}\right) \cap X\right)$. But by Lemma $3 \nu(T E)=\int_{E} \lambda e^{-\varphi(y)} d \nu(y)=$ 0 .

To show $T$ is nonsingular it suffices to show that if $F \subset B_{2 e_{0}}\left(x_{j}\right) \cap X$ and $\nu(F)=0$ then $\nu\left(T^{-1} F \cap A_{i}\left(x_{j}\right)\right)=0$ for all components $A_{i}\left(x_{j}\right)$ of $T^{-1}\left(B_{2 e_{0}}\left(x_{j}\right) \cap X\right)$. But

$$
0=\nu(F)=\int_{T^{-1} F \cap A_{i}\left(x_{j}\right)} \lambda e^{-\varphi(y)} d \nu(y)
$$

by Lemma 3 and so $\nu\left(T^{-1} F \cap A_{i}\left(x_{j}\right)\right)=0$.

To prove the convergence theorems we require one more assumption on $T$ and one more on $\varphi$. The following is a mixing condition on $T$. 
II. $\forall \varepsilon>0, \exists M>0$ such that for each $x \in X, T^{-M} x$ is $\varepsilon$-dense in $X$. (We stress that $M$ is independent of $x$.)

The following condition gives a further restriction on $\varphi$.

(iii) If $d\left(x, x^{\prime}\right)<\varepsilon_{0}$ then

$$
C_{\varphi}\left(x, x^{\prime}\right)=\sup _{n>1} \sup _{y \in T^{-n_{x}}} \sum_{i=0}^{n-1}\left[\varphi\left(T^{i} y\right)-\varphi\left(T^{i} y^{\prime}\right)\right]
$$

exists, is bounded above by a constant $C_{\varphi}$, and $C_{\varphi}\left(x, x^{\prime}\right) \rightarrow 0$ as $d\left(x, x^{\prime}\right) \rightarrow 0$.

We make some remarks about condition (iii).

Remarks. (a) For $n \geqslant 1$ and $y \in T^{-n} x$ we have

$$
-C_{\varphi}\left(x^{\prime}, x\right)<\sum_{i=0}^{n-1}\left[\varphi\left(T^{i} y\right)-\varphi\left(T^{\prime} \dot{y}^{\prime}\right)\right]<C_{\varphi}\left(x, x^{\prime}\right),
$$

so that condition (iii) is equivalent to the following statement;

$$
\sup _{n>1} \sup _{y \in T^{-n_{x}}}\left|\sum_{i=0}^{n-1}\left[\varphi\left(T^{\dot{y}}\right)-\varphi\left(T^{\dot{y}^{\prime}}\right)\right]\right| \leqslant C_{\varphi} \quad \text { whenever } d\left(x, x^{\prime}\right)<\varepsilon_{0}
$$

and

$$
\sup _{n>1} \sup _{y \in T^{-n_{x}}}\left|\sum_{i=0}^{n-1}\left[\varphi\left(T^{\dot{y}} y\right)-\varphi\left(T^{\dot{y}^{\prime}}\right)\right]\right| \rightarrow 0 \text { as } d\left(x, x^{\prime}\right) \rightarrow 0 .
$$

(b) If $C_{i}$ is a component of $T^{-n}\left(B_{\varepsilon_{0}}(x) \cap X\right)$ (i.e., $T^{n}$ maps $C_{i}$ onto $B_{\varepsilon_{0}}(x) \cap X$ not decreasing distances) there exists $d_{i}$ such that $\left|\left(S_{n} \varphi\right)(y)\right|<d_{i}$ $\forall y \in C_{i}$. This is because for any fixed $y^{\prime} \in C_{i}$

$$
\begin{aligned}
\left|S_{n} \varphi(y)\right| & <\left|S_{n} \varphi(y)-S_{n} \varphi\left(y^{\prime}\right)\right|+\left|S_{n} \varphi\left(y^{\prime}\right)\right| \\
& <C_{\varphi}+\left|S_{n} \varphi\left(y^{\prime}\right)\right|=d_{i} .
\end{aligned}
$$

(c) Conditions (i) and (iii) imply condition (ii). This is because if $x, x^{\prime} \in X$ and $d\left(x, x^{\prime}\right)<\varepsilon_{0}$ then

$$
\begin{aligned}
\sum_{y \in T^{-1} x}\left|e^{\varphi(y)}-e^{\varphi\left(y^{\prime}\right)}\right| & =\sum_{y \in T^{-1} x} e^{\varphi\left(y^{\prime}\right)}\left|e^{\varphi(y)-\varphi\left(y^{\prime}\right)}-1\right| \\
& <\sum_{y \in T^{-1} x} e^{\varphi\left(y^{\prime}\right)} \max \left[e^{C_{\varphi}\left(x, x^{\prime}\right)}-1,1-e^{-C_{\varphi}\left(x^{\prime}, x\right)}\right] \\
& <K \max \left[e^{C_{\varphi}\left(x, x^{\prime}\right)}-1,1-e^{-C_{\varphi}(x, x)}\right] .
\end{aligned}
$$

(d) The conditions II, (i), (ii), (iii) do not depend on the choice of the metric $d$ on $\bar{X}$. Condition I depends on $d$ and in some examples we can change to an equivalent metric for which I holds.

An important consequence of II and (iii) is the following:

LEMMA 5. Let $T: X_{0} \rightarrow X$ satisfy I and II and let $\varphi \in C(X)$ satisfy (i) and 
(iii). Then $\forall \varepsilon>0, \exists N>0$ and $a \in R$ such that $\forall x, w \in X \exists y \in T^{-N} x \cap$ $B_{e}(w)$ with $\sum_{i=1}^{N-1} \varphi\left(T^{i} y\right)>a$.

Proof. Let $\varepsilon<\varepsilon_{0}$ be given. Using II choose $N$ so that $T^{-N} x$ is $\varepsilon / 4$ dense in $X$ for each $x \in X$. Choose $w_{1}, \ldots, w_{r}$ so that $B_{\varepsilon / 2}\left(w_{j}\right)$ cover $\bar{X}$. It suffices to choose $a_{j} \in R(1<j<r)$ so that $\forall x \in X \exists y \in T^{-N} x \cap B_{e / 2}\left(w_{j}\right)$ with $S_{N} \varphi(y)>a_{j}$; because then we can take $a=\min _{1<j<r} a_{j}$. Choose $x_{1}, \ldots, x_{m}$ so that $B_{\varepsilon / 4}\left(x_{i}\right)$ cover $\bar{X}$. Fix $j$. Since $T^{-N} x_{i}$ is $\varepsilon / 4$ dense $\exists y_{i} \in T^{-N} x_{i}$ with $d\left(y_{i}, w_{j}\right)<\varepsilon / 4$. Let $x \in X$. Choose $i$ so that $d\left(x, x_{i}\right)<\varepsilon / 4$. Let $y$ be the point of $T^{-N} x$ corresponding to $y_{i}$. Then

$$
d\left(y, w_{j}\right)<d\left(y, y_{i}\right)+d\left(y_{i}, w_{j}\right)<\varepsilon / 4+\varepsilon / 4=\varepsilon / 2
$$

so $y \in T^{-N^{N}} \cap B_{\varepsilon / 2}\left(w_{j}\right)$. Also

$$
\begin{aligned}
S_{N} \varphi(y) & =S_{N} \varphi(y)-S_{N} \varphi\left(y_{i}\right)+S_{N} \varphi\left(y_{i}\right) \\
& >S_{N} \varphi\left(y_{i}\right)-C_{\varphi} \\
& >\min _{i}\left[S_{N} \varphi\left(y_{i}\right)-C_{\varphi}\right] \equiv a_{j} .
\end{aligned}
$$

We shall be especially interested in a certain subclass of $C\left(X_{0}\right)$. Let $G\left(X_{0}\right)=\left\{g \in C\left(X_{0}\right) \mid g>0\right.$ and $\left.\Sigma_{y \in T^{-1} x} g(y)=1 \forall x \in X\right\}$. For $\varphi=\log g$ condition (i) holds with $K=1$, and condition (ii) becomes

(ii) ${ }_{G}$

$$
\sum_{y \in T^{-1} x}\left|g(y)-g\left(y^{\prime}\right)\right| \rightarrow 0 \text { as } d\left(x, x^{\prime}\right) \rightarrow 0 .
$$

If (ii) $)_{G}$ is satisfied then $\mathfrak{L}_{\log g}: U C(X) \rightarrow U C(X)$ and $\overline{\mathrm{e}}_{\log g}: C(\bar{X}) \rightarrow C(\bar{X})$ are defined. Condition (iii) becomes

(iii) ${ }_{G}$ If $d\left(x, x^{\prime}\right)<\varepsilon_{0}$ then

$$
D_{g}\left(x, x^{\prime}\right)=\sup _{n>1} \sup _{y \in T^{-n_{x}}} \prod_{i=0}^{n-1} \frac{g\left(T^{i} y\right)}{g\left(T^{i} y^{\prime}\right)}
$$

exists, is bounded above by $D_{g}$, and $D_{g}\left(x, x^{\prime}\right) \rightarrow 1$ as $d\left(x, x^{\prime}\right) \rightarrow 0$. Equivalently:

$$
D_{g}^{*}\left(x, x^{\prime}\right)=\sup _{n>1} \sup _{y \in T^{-n} x}\left|\prod_{i=0}^{n-1} \frac{g\left(T^{\dot{y}}\right)}{g\left(T^{i} y^{\prime}\right)}-1\right| \leqslant D_{g}-1
$$

for all $x, x^{\prime}$ with $d\left(x, x^{\prime}\right)<\varepsilon_{0}$ and $D_{g}^{*}\left(x, x^{\prime}\right) \rightarrow 0$ as $d\left(x, x^{\prime}\right) \rightarrow 0$. By remark (c) above (iii) ${ }_{G}$ implies (ii) $)_{G}$.

If $T$ satisfies I and II and $g \in G\left(X_{0}\right)$ satisfies (iii) $)_{G}$ then Lemma 5 asserts that $\forall \varepsilon>0, \exists N>0$ and $b>0$ such that $\forall x, w \in X \exists y \in T^{-N} x \cap B_{\varepsilon}(w)$ with $\prod_{i=0}^{N-1} g\left(T^{i} y\right) \geqslant b>0$.

The following result appeared in [10] for the case of a shift space on a finite alphabet (with a stronger assumption on $g$ ) and in [24] for the case of subshifts of finite type. 
Theorem 6. Let $T: X_{0} \rightarrow X$ satisfy I and II and let $g \in G\left(X_{0}\right)$ satisfy (iii) ${ }_{G}$. Then there exists $\mu \in M(\bar{X})$ such that $\overline{\mathrm{L}}_{\log g}^{n} f \rightarrow \mu(f) \forall f \in C(\bar{X})$. $\mu$ is the only member of $M(\bar{X})$ with $\overline{\mathrm{C}}_{\log g}^{*} \mu=\mu$.

Proof. Write $\overline{\mathfrak{L}}$ instead of $\overline{\mathfrak{L}}_{\text {log g }}$. Let $f \in C(\bar{X})$. We show $\left\{\overline{\mathfrak{L}}^{n} f \mid n>0\right\}$ is equicontinuous. Let $x, x^{\prime} \in X$ and $d\left(x, x^{\prime}\right)<\varepsilon<\varepsilon_{0}$. Then

$$
\begin{aligned}
& \left|\overline{\mathfrak{L}} n f(x)-\overline{\mathfrak{L}^{n}} f\left(x^{\prime}\right)\right| \\
& <\left|\sum_{y \in T^{-n_{x}}} g(y) g(T y) \cdots g\left(T^{n-1} y\right)\left[f(y)-f\left(y^{\prime}\right)\right]\right| \\
& +\mid \sum_{y \in T^{-n_{x}}} f\left(y^{\prime}\right)\left[g(y) g(T y) \cdots g\left(T^{n-1} y\right)\right. \\
& \left.-g\left(y^{\prime}\right) g\left(T y^{\prime}\right) \cdots g\left(T^{n-1} y^{\prime}\right)\right] \\
& <\sup \{\mid f(u)-f(v) \| d(u, v)<\varepsilon\} \\
& +\|f\| \sum_{y \in T^{-n_{x}}} g\left(y^{\prime}\right) \cdots g\left(T^{n-1} y^{\prime}\right)\left|\frac{g(y) \cdots g\left(T^{n-1} y\right)}{g\left(y^{\prime}\right) \cdots g\left(T^{n-1} y^{\prime}\right)}-1\right| \\
& <\sup \{\mid f(u)-f(v) \| d(u, v)<\varepsilon\}+\|f\| D_{\varepsilon}^{*}\left(x, x^{\prime}\right) .
\end{aligned}
$$

Therefore $\left\{\overline{\mathfrak{L}}^{n} f \mid n>0\right\}$ is equicontinuous. Since $\left\|\overline{\mathfrak{L}}^{n} f\right\|<\|f\|, f \in C(\bar{X})$, we know the closure of $\left\{\overline{\mathrm{L}}^{n} f \mid n>0\right\}$ in $C(\bar{X})$ is compact. Hence there is a sequence $n_{i} \rightarrow \infty$ and $f^{*} \in C(\bar{X})$ with $\overline{\mathfrak{L}}^{n_{i}} f \rightarrow f^{*}$. We have

$$
\begin{aligned}
\min (f) & <\min (\overline{\mathscr{L}} f)<\cdots<\min \left(f^{*}\right)<\max \left(f^{*}\right) \\
& <\cdots<\max (\overline{\mathscr{L}} f)<\max (f) .
\end{aligned}
$$

Clearly $\min \left(\mathfrak{L}^{k} f^{*}\right)=\min \left(f^{*}\right)$ for all $k>0$. We want to $\operatorname{show} \min \left(f^{*}\right)=f^{*}$ and to do this we shall show that if $\varepsilon>0$ and $\delta>0$ then $f^{*}(u)<\min \left(f^{*}\right)+$ $\delta$ for all points $u$ in an $\varepsilon$-dense subset of $X$. Choose $N>0$ and $b>0$ so that $\forall x, w \in X \quad \exists y \in T^{-N} x \cap B_{e}(w)$ with $\prod_{i=0}^{N-1} g\left(T^{i} y\right)>b$ (by Lemma 5). Choose $z \in \bar{X}$ with $\min \left(\overline{\mathfrak{L}}^{N} f^{*}\right)=\overline{\mathfrak{L}}^{N} f^{*}(z)$. If $z \in X$ then $f^{*}(y)=\min \left(f^{*}\right)$ $\forall y \in T^{-N_{z}}$ and so $f^{*}(y)=\min \left(f^{*}\right)$ for points $y$ in an $\varepsilon$-dense set. Suppose that $z \notin X$. We shall show that if $x \in X$ is sufficiently close to $z$ and if $\Pi_{i=0}^{N-1} g\left(T^{\dot{y}}\right)>b$ for some $y \in T^{-N} x$ then $f^{*}(y)<\min \left(f^{*}\right)+\delta$. Suppose $y \in T^{-N} x$ is such that $\prod_{i=0}^{N-1} g\left(T^{\dot{y}}\right)>b$ but $f^{*}(y)>\min \left(f^{*}\right)+\delta$. Then

$$
\begin{aligned}
\overline{\mathfrak{e}}^{N} f^{*}(x)= & \sum_{u \in T^{-N_{x}}} g(u) \cdots g\left(T^{N-1} u\right) f^{*}(u) \\
& >\min \left(f^{*}\right)+g(y) \cdots g\left(T^{N-1} y\right) \delta \geqslant \min \left(f^{*}\right)+b \delta,
\end{aligned}
$$


and this does not happen if $x$ is close enough to $z$.

Therefore $\min \left(f^{*}\right)=f^{*}$. Put $\mu(f)=f^{*}$. Then $\mu: C(\bar{X}) \rightarrow R$ is a member of $M(\bar{X})$ by the Riesz representation theorem. Clearly $\mathcal{L}^{*} \mu=\mu$. If $m \in M(\bar{X})$ and $\overline{\mathfrak{L}}^{*} m=m$ then integrating $\overline{\mathfrak{L}}^{n} f \Rightarrow \mu(f)$ with respect to $m$ gives $m(f)=$ $\mu(f) \forall f \in C(\bar{X})$. Hence $m=\mu$.

If $f: X_{0} \rightarrow R$ is not continuous we can still write

$$
\mathfrak{L}_{\log g} f(x)=\sum_{y \in T^{-1} x} g(y) f(y), \quad x \in X .
$$

We shall use the following corollary when investigating Bernoulli properties of $T$.

COROLlary 7. Let $T$ and $g$ be as in Theorem 6 and let $\mu$ be the measure obtained in that theorem. If $\mu(\partial B)=0$ then

$$
\sup _{x \in X}\left|\mathbb{R}^{n} \chi_{B}(x)-\mu(B)\right| \rightarrow 0 \text { as } n \rightarrow \infty \text {. }
$$

Proof. Let $\varepsilon>0$ be given. Choose an open set $U$ with $\bar{B} \subset U$ and $\mu(U \backslash B)=\mu(U \backslash \bar{B})<\varepsilon$. Let $f_{+} \in C(\bar{X})$ be chosen so that $f_{+}=1$ on $\bar{B}$, $0<f_{+}<1$, and $f_{+}=0$ on $\bar{X} \backslash U$. Also choose a compact set $C \subset \operatorname{int}(B)$ such that $\mu(B \backslash C)=\mu(\operatorname{int}(B) \backslash C)<\varepsilon$ and construct $f_{-} \in C(\bar{X})$ so that $f_{-}=1$ on $C, 0<f_{-}<1$, and $f_{-}=0$ on $\bar{X} \backslash \operatorname{int}(B)$. Then $f_{-}<\chi_{B}<f_{+}$ and $\mu\left(f_{+}\right)-\mu(B)<\varepsilon, \mu(B)-\mu\left(f_{-}\right)<\varepsilon$. Therefore

$$
\mathfrak{L}^{n} f_{-}(x) \leqslant \mathfrak{L}^{n} \chi_{B}(x) \leqslant \mathfrak{E}^{n} f_{+}(x) \text { for } x \in X
$$

and

$$
\begin{array}{r}
\left|\mathfrak{L}^{n} \chi_{B}(x)-\mu(B)\right|<\max \left(\left|\mathbb{L}^{n} f_{+}(x)-\mu\left(f_{+}\right)\right|+\mu\left(f_{+}\right)-\mu(B),\right. \\
\left.\left|\mathfrak{L}^{n} f_{-}(x)-\mu\left(f_{-}\right)\right|+\mu(B)-\mu\left(f_{-}\right)\right) .
\end{array}
$$

We can choose $N$ by Theorem 6 so that $n \geqslant N$ implies

$$
\sup _{x \in X}\left|\mathscr{L}^{n} \chi_{B}(x)-\mu(B)\right| \leqslant 2 \varepsilon
$$

Theorem 6 will be used to prove the following theorem which is the main result of this paper. It was proved by Ruelle when $T$ is the one-sided shift on an alphabet of two points [20] and by Bowen for a general mixing subshift of finite type [3], [5]. That case is also considered in [24] with the same scheme of proof as we use here.

THeOREM 8. Let $T: X_{0} \rightarrow X$ satisfy I and II. Let $\varphi \in C\left(X_{0}\right)$ satisfy (i) and (iii). Let $\nu \in M(\bar{X})$ and $\lambda>0$ be such that $\overline{\mathfrak{L}}^{*} \nu=\lambda \nu$ ( $\nu$ and $\lambda$ exist by Lemma 2). Then

(a) $\exists h \in C(\bar{X}), h>0$ such that $\nu(h)=1, \mathfrak{\ell}_{\varphi} h=\lambda h$ and

$$
\frac{1}{\lambda^{n}} \overline{\mathfrak{e}}_{\varphi}^{n} f \Rightarrow h \cdot \nu(f) \quad \forall f \in C(\bar{X}) \text {. }
$$


(b) The function $g=\left(e^{\varphi} \cdot h\right) /(\lambda \cdot h \circ T)$ belongs to $G\left(X_{0}\right)$, satisfies (iii) $G$, and the measure $\mu$ determined by Theorem 6 is $h \cdot \nu$. (Therefore $\mu$ and $\nu$ are equivalent.)

(c) The pair $(\lambda, \nu)$ is uniquely determined by the conditions $\lambda>0, \nu \in M(\bar{X})$ and $\overline{\mathrm{E}}_{\phi}^{*} \nu=\lambda \nu$.

(d) $h$ satisfies $h(x)<e^{C_{\nabla}\left(x, x^{\prime}\right)} h\left(x^{\prime}\right)$ if $d\left(x, x^{\prime}\right)<\varepsilon_{0} . h$ is uniquely determined by this condition and the properties $h>0, \nu(h)=1$ and $\overline{\mathfrak{e}}_{\varphi} h=\lambda h$.

Proof. We shall write $\mathcal{L}$ instead of $\mathcal{E}_{\varphi}$ and $C, C\left(x, x^{\prime}\right)$ instead of $C_{\varphi}$, $C_{\varphi}\left(x, x^{\prime}\right)$. We first show the existence of $h$. Let

$$
\begin{aligned}
\Lambda=\{f \in C(\bar{X}) \mid f>0, \nu(f) & =1 \text { and } f(x)<e^{C\left(x, x^{\prime}\right)} f\left(x^{\prime}\right) \\
& \text { if } \left.x, x^{\prime} \in X \text { and } d\left(x, x^{\prime}\right)<\varepsilon_{0}\right\} .
\end{aligned}
$$

$\Lambda$ is nonempty because we can show $\lambda^{-1} \overline{\mathscr{L}} 1 \in \Lambda$ as follows. Clearly, this function is nonnegative and has integral 1 , and if $x, x^{\prime} \in X$ and $d\left(x, x^{\prime}\right)<\varepsilon_{0}$ then

$$
\begin{aligned}
\overline{\mathfrak{L}}_{1} 1(x) & =\sum_{y \in T^{-1} x} e^{\varphi(y)} \leqslant \exp \left(\sup _{y \in T^{-1} x}\left[\varphi(y)-\varphi\left(y^{\prime}\right)\right]\right) \sum_{y^{\prime} \in T^{-1} x^{\prime}} e^{\varphi\left(y^{\prime}\right)} \\
& <e^{C\left(x_{0} x^{\prime}\right) \bar{L}} 1\left(x^{\prime}\right) .
\end{aligned}
$$

$\Lambda$ is clearly convex and closed. We shall show it is also bounded and equicontinuous.

Fix some $\varepsilon_{1}<\varepsilon_{0}$. Let $N$ and $a$ be chosen for $\varepsilon_{1}$ by Lemma 5. Let $x, w \in X$. Choose some point $y_{0} \in T^{-N} x \cap B_{\varepsilon_{1}}(w)$ with $S_{N} \varphi\left(y_{0}\right)>a$. Then

$$
\begin{aligned}
\overline{\mathfrak{L}}^{N} f(x) & =\sum_{y \in T^{-N_{x}}} e^{S_{N} \varphi(y)} f(y)>e^{S_{N} \varphi\left(y_{0}\right)} f\left(y_{0}\right) \\
& >e^{a} f\left(y_{0}\right)>e^{a-C} f(w) .
\end{aligned}
$$

Therefore $f(w)<e^{c-a \overline{\mathfrak{e}}^{N}} f(x)$ for all $w, x \in X$ and hence for all $w, x \in \bar{X}$. This gives $f(w)<e^{c-a} \nu\left(\overline{\mathscr{E}}^{N} f\right)=e^{c-a} \lambda^{N}=Q$ for all $w \in \bar{X}$. Hence $\Lambda$ is bounded. then

$\Lambda$ is equicontinuous because if $f \in \Lambda$ and $x, x^{\prime} \in X$ satisfy $d\left(x, x^{\prime}\right)<\varepsilon_{0}$

$$
\begin{aligned}
\left|f(x)-f\left(x^{\prime}\right)\right| & =\max \left(f(x)-f\left(x^{\prime}\right), f\left(x^{\prime}\right)-f(x)\right) \\
& <\max \left(f\left(x^{\prime}\right)\left[e^{C\left(x, x^{\prime}\right)}-1\right], f(x)\left[e^{C\left(x^{\prime}, x\right)}-1\right]\right) \\
& <Q \max \left(e^{C\left(x, x^{\prime}\right)}-1, e^{C\left(x^{\prime}, x\right)}-1\right),
\end{aligned}
$$

and this is small when $d\left(x, x^{\prime}\right)$ is small, by condition (iii).

Therefore $\Lambda$ is a nonempty, compact, convex subset of $C(\bar{X})$. We now show that $\lambda^{-1} \overline{\mathcal{L}}$ maps $\Lambda$ into $\Lambda$. If $f \in \Lambda$ and $x, x^{\prime} \in X, d\left(x, x^{\prime}\right)<\varepsilon_{0}$ then 


$$
\begin{aligned}
\frac{1}{\lambda} \bar{\varrho} f(x) & =\frac{1}{\lambda} \sum_{y \in T^{-1} x} e^{\varphi(y)} f(y) \\
& <\frac{1}{\lambda} \sum_{y \in T^{-1} x} e^{\varphi\left(y^{\prime}\right)} f\left(y^{\prime}\right)\left(e^{\varphi(y)-\varphi\left(y^{\prime}\right)} e^{c\left(y y^{\prime}\right)}\right) \\
& <\frac{1}{\lambda} \bar{\varrho} f\left(x^{\prime}\right) e^{c\left(x, x^{\prime}\right)} .
\end{aligned}
$$

We can now apply the Schauder-Tychonoff fixed point theorem to obtain a fixed point $h \in \Lambda$ for $\lambda^{-1} \overline{\mathcal{L}}$. Therefore $\overline{\mathrm{L}} h=\lambda h, \nu(h)=1, h>0$ and $h(x)<$ $e^{c\left(x, x^{\prime}\right)} h\left(x^{\prime}\right)$ if $d\left(x, x^{\prime}\right)<\varepsilon_{0}$. We now show $h>0$. If $h(x)=0$ for some $x \in X$ then since $\mathbb{P}^{n} h(x)=\lambda^{n} h(x)=0$ we conclude that $h$ vanishes on the set $\cup_{n=0}^{\infty} T^{-n} x$, which is dense by condition II. Then $h \equiv 0$ contradicting $\nu(h)=$ 1. Therefore $h>0$ on $X$. Let $x \in \bar{X}$. The ball $B_{\varepsilon_{0} / 2}(w)$ contains points $x_{n}$ of $X$ which converge to $w$ and they satisfy $h\left(x^{\prime}\right)<e^{C} h\left(x_{n}\right)$ for any point $x^{\prime} \in X$ $\cap B_{\varepsilon_{0} / 2}(w)$. Then $h(w)>e^{-C_{h}}\left(x^{\prime}\right)>0$.

Put $g=e^{\varphi} h /\left(\lambda_{h} \circ T\right)$. Clearly $g \in G\left(X_{0}\right)$. We show $g$ satisfies (iii) $G$. Let $x$, $x^{\prime} \in X$ and $d\left(x, x^{\prime}\right)<\varepsilon_{0}$. If $y \in T^{-n} x$ then

$$
\prod_{i=0}^{n-1} \frac{g\left(T^{\dot{y}}\right)}{g\left(T^{i} y^{\prime}\right)}=e^{S_{n} \varphi(y)-S_{n} \varphi\left(y^{\prime}\right)} \frac{h(y)}{h\left(y^{\prime}\right)} \frac{h\left(x^{\prime}\right)}{h(x)}
$$

so that

$$
\begin{aligned}
e^{S_{n} \varphi(y)-S_{n} \varphi\left(y^{\prime}\right)-C\left(y^{\prime} y\right)-C\left(x, x^{\prime}\right)} & <\prod_{i=0}^{n-1} \frac{g(T \dot{y})}{g\left(T^{\prime} y^{\prime}\right)} \\
& <e^{S_{n} \varphi(y)-S_{n} \varphi\left(y^{\prime}\right)+C\left(y, y^{\prime}\right)+C\left(x^{\prime}, x\right)}
\end{aligned}
$$

and hence

$$
e^{-C\left(x, x^{\prime}\right)-C\left(x^{\prime}, x\right)}<\prod_{i=0}^{n-1} \frac{g(T \dot{y})}{g\left(T^{i} y^{\prime}\right)}<e^{C\left(x, x^{\prime}\right)+C\left(x^{\prime}, x\right)}
$$

Therefore (iii) ${ }_{G}$ is satisfied.

By Theorem 6 we have $\bar{l}_{\log g}^{n} \Rightarrow \mu(f) \forall f \in C(\bar{X})$ where $\mu \in M(\bar{X})$ is the only fixed point of $\overline{\mathrm{L}}_{\log 8}^{*}$ in $M(\bar{X})$. We have

$$
\frac{1}{\lambda^{n}}\left(\overline{\mathcal{L}}_{\varphi}^{n} f\right)(x)=h(x)\left(\overline{\tilde{E}}_{\log g}^{n} f / h\right)(x)
$$

so that $\left(1 / \lambda^{n}\right) \overline{\mathcal{E}}_{\varphi}^{n} f \Rightarrow h \cdot \nu(f / h)$. We want to show $\mu(f / h)=\nu(f)$. Let $m \in$ $M(\bar{X})$ be defined by $m(f)=\nu(h f)$. Then

$$
m\left(\overline{\mathscr{E}}_{\log 8} f\right)=\nu\left(h \cdot \overline{\mathscr{L}}_{\log g} f\right)=\frac{1}{\lambda} \nu\left(\overline{\mathcal{E}}_{\varphi}(f \cdot h)\right)=\nu(f \cdot h)=m(f)
$$

so that $m=\mu$. Therefore $\mu(f)=\nu(h f)$.

It remains to check (c). This follows because by (a) we get 


$$
\log \lambda=\lim _{n \rightarrow \infty} \frac{1}{n} \log \overline{\mathfrak{e}}_{\varphi}^{n} 1 \text { and } \nu(f)=\lim _{n \rightarrow \infty} \cdot \frac{\overline{\mathfrak{e}}_{\varphi}^{n} f}{\overline{\mathfrak{e}}_{\varphi}^{n} 1} .
$$

Some Corollaries of this theorem will be stated later (Corollary 12).

Given $\varphi \in C\left(X_{0}\right)$ satisfying (i) and (iii) we shall use the notation $\lambda, \nu, h, g$, $\mu$ to denote the objects given by Theorems 6 and 8 . If we wish to emphasize the dependence on $\varphi$ we will add a subscript $\varphi$.

So far we have not used the fact that $X$ is an open subset of $\bar{X}$ but we use this in part (2) of the next result where we show $\mu$ (and therefore $\nu$ ) is concentrated on $X_{0}$. When $X=\bar{X}$ this follows immediately from part (1). In the problem of invariant measures the measure $\nu$ is given and so part (2) is redundant in that case. It is only needed for the case of equilibrium states when $X \neq \bar{X}$. If we know that $\mu_{\varphi}\left(X_{0}\right)=1$ then the results of the paper are true for $\mu_{\varphi}$ when $X$ is a (not necessarily open) dense subset of $\bar{X}$ and $X_{0}$ is an open and dense subset of $X$. However we need to assume $X$ open to get $\mu\left(X_{0}\right)=1$ for all the functions satisfying conditions (i) and (iii).

Leman 9. Let $T: X_{0} \rightarrow X$ satisfy I and II and let $\varphi$ satisfy (i) and (iii). Then

(1) $T: X_{0} \rightarrow X$ is measure preserving relative to the measures $\mu \mid X_{0}$ and $\mu \mid X$ (i.e., if $B \in X$ then $\left.\mu\left(T^{-1} B\right)=\mu(B)\right)$.

(2) $\mu\left(X_{0}\right)=\nu\left(X_{0}\right)=1$.

Proor. (1) We shall show that if $f \in C(X)$ is nonnegative and has compact support inside some $X \cap B_{2 e_{0}}(x)$ then $\int f T d \mu=\int f d \mu$. Let the components of $T^{-1}\left(X \cap B_{2 \varepsilon_{0}}(x)\right)$ be $A_{1}, A_{2}, \ldots$ and let $T_{i}=\left.T\right|_{A_{i}}$. Then $f \circ T_{i}$ has compact support inside $A_{i}$ and we can consider it as defined on all of $\bar{X}$ by putting it zero outside $A_{i}$. Then $f(T z)=\Sigma_{i} f\left(T_{i} z\right)$ and

$$
\int f T_{i} d \mu=\int \overline{\mathrm{e}}_{\log g}\left(f T_{i}\right) d \mu=\int_{X \cap B_{22_{0}}(x)} g\left(T_{i}^{-1}(x)\right) f(x) d \mu(x) .
$$

Summing this equation gives $\int f T d \mu=\int f d \mu$.

(2) By (1) we know $\mu\left(X_{0}\right)=\mu(X)$ so we have to show $\mu(\partial X)=0$, or equivalently $\nu(\partial X)=0$.

Choose $\varepsilon_{1}>0$ so that $\varepsilon_{1}<\varepsilon_{0}$ and so that $X$ contains a ball of radius $4 \varepsilon_{1}$. Choose $N$ so that $T^{-N} x$ is $\varepsilon_{1}$-dense for all $x$. Let $U_{\delta}$ be the $\delta$-ball of $\partial X$ i.e., $U_{\delta}=\{x \in \bar{X} \mid d(x, \partial X)<\delta\}$. Since $\partial X$ is compact choose a finite cover $V_{1}, \ldots, V_{r}$ of $\partial X$ by open balls of radius $\varepsilon_{1}$. Let $V_{i}(\delta)=U_{\delta} \cap V_{i}, 1<i<r$. Then $V_{i}(\delta), \ldots, V_{r}(\delta)$ is an open cover of $\partial X$ and as $\delta \rightarrow 0 \cup_{i=1}^{r} V_{i}(\delta)$ decreases to $\partial X$. Since $V_{i}(\delta) \subset V_{i}$, condition I implies $T^{-N}\left(V_{i}(\delta) \cap X\right)$ is a disjoint union of open sets of diameter less then $2 \varepsilon_{1}$. Since $T^{-N} X$ is $\varepsilon_{1}$-dense, one of these open sets, say $E_{i}(\delta)$, has distance greater than $\varepsilon_{1}$ from $\partial X$. We can choose the sets $E_{i}(\delta)$ to decrease as $\delta$ decreases. Consider $\cap_{\delta>0} \overline{E_{i}(\delta)}=$ $\Omega . \Omega$ is disjoint from $\partial X$ by choice of $E_{i}(\delta)$, and we now show that 
$\Omega \subset X \backslash T^{-N} X$. If not then let $x \in \Omega \cap T^{-N} X$. Then

$$
T^{N} x \in \overline{V_{i}(\delta)} \cap X \subset \bar{U}_{\delta} \cap \bar{V}_{i} \cap X
$$

for all $\delta>0$ and so $T^{N} x \in \partial X \cap \bar{V}_{i} \cap X=\varnothing$, a contradiction. Therefore

$$
\Omega \subset X \backslash T^{-N} X=\bigcap_{j=0}^{N-1} T^{-j}\left(X \backslash T^{-1} X\right)
$$

which is a null set by part (1). Hence $\nu(\Omega)=0$. This implies $\nu\left(\bar{E}_{i}(\delta)\right) \rightarrow 0$ as $\delta \rightarrow 0$. Fix $\delta>0$. Let $\varepsilon>0$ be given and choose an open set $U \supset \bar{E}_{i}(\delta)$ with $\nu\left(U \backslash \bar{E}_{i}(\delta)\right)<\varepsilon$. Let $f \in C(\bar{X})$ be so that $f=1$ on $\bar{E}_{i}(\delta), 0<f<1$, and $f=0$ outside $U$. Then

$$
\begin{aligned}
\lambda^{N}\left[\nu\left(\bar{E}_{i}(\delta)\right)+\varepsilon\right] & >\lambda^{N} \int f d \nu=\int \overline{\mathcal{E}}_{\varphi}^{N} f d \nu \\
& \geqslant\left[\inf _{y \in E_{i}(\delta)} e^{S_{N} \varphi(\nu)}\right] \nu\left(\partial X \cap V_{i}(\delta)\right) \\
& =\left[\inf _{y \in E_{i}(\delta)} e^{S_{N} \varphi(\nu)}\right] \nu\left(\partial X \cap V_{i}\right) .
\end{aligned}
$$

Therefore $\lambda^{N} \nu\left(\bar{E}_{i}(\delta)\right)>d_{i} \nu\left(\partial X \cap V_{i}\right)$ for some constant $d_{i}$ (see remark (b) after the statement of condition (iii)) and when $\delta \rightarrow 0$ we get $\nu\left(\partial X \cap V_{i}\right)=0$. Hence $\nu(\partial X)=0$.

We now know the measures $\mu_{\varphi}$ we are interested in are concentrated on $X_{0}$. We now look at equivalent ways of describing measures that have the property $\overline{\mathcal{E}}_{\log 8}^{*} \mu=\mu$ for some $g \in G\left(X_{0}\right)$. This next theorem generalizes the one given in [12] for shift systems. Even though $X$ is not compact if $X \neq \bar{X}$ we shall still use $M(X)$ to denote the collection of all probability measures defined on the Borel subsets $\mathscr{B}$ of $X$, and $M(X, T)$ to denote the $T$-invariant ones. If $\mathcal{C}$ is a subalgebra of $\mathscr{B}$ and $m \in M(X)$ then $E_{m}(f / \mathcal{C})$ will denote the conditional expectation of $f$ with respect to $C$; and $I_{m}(\mathscr{G} / \mathcal{C})$ will denote the conditional information of $\mathscr{B}$ with respect to $C$.

Theorem 10. Let $T$ satisfy I and let $g \in G\left(X_{0}\right)$ satisfy (ii) ${ }_{G}$. Let $\sigma \in M(X)$. The following are equivalent:

(1) $\overline{\mathrm{E}}_{\log 8}^{*} \sigma=\sigma$.

(2) $\sigma \in M(X, T)$ and

$$
E_{o}\left(f / T^{-1} \mathscr{B}\right)(x)=\sum_{y \in T^{-1} x} g(y) f(y) \text { a.e. }(\sigma) \quad \forall f \in C(\bar{X}) .
$$

(3) $\sigma \in M(X, T)$ and

$$
\sigma\left(I_{\sigma}\left(\mathscr{B} / T^{-1} \mathscr{B}\right)+\log g\right)>m\left(I_{m}\left(\mathscr{B} / T^{-1} \mathscr{B}\right)+\log g\right) \quad \forall m \in M(X, T) .
$$

Proof. We shall write $\mathcal{L}$ instead of $\mathscr{L}_{\text {log } g}$. The proof that (1) implies $\sigma \in M(X, T)$ is exactly the same as the proof of part (1) of Lemma 9. Let 
$f \in C(\bar{X})$. Then

$$
\int f d \sigma=\int \varrho f d \sigma=\int \mathscr{E} f d \sigma=\int(\mathscr{L} f) \cdot T d \sigma=\int \sum_{y \in T^{-1} T x} g(y) f(y) d \sigma(x) .
$$

Therefore

$$
E_{o}\left(f / T^{-1} \mathscr{B}\right)(x)=\sum_{y \in T^{-1} T x} g(y) f(y) \text { a.e. (o). }
$$

Now assume (2) holds. Then $I_{\sigma}\left(\mathscr{B} / T^{-1} \mathscr{B}\right)=-\log g$. Let $m \in M(X, T)$ and let $g_{m}: X_{0} \rightarrow R$ be the function defined a.e. $(m)$ by

$$
E_{m}\left(f / T^{-1 \mathscr{B}}\right)(x)=\sum_{y \in T^{-1} T x} g_{m}(y) f(y) .
$$

$I_{m}\left(\mathscr{B} / T^{-1} \mathscr{B}\right)=-\log g_{m}$, therefore

$$
\begin{aligned}
m\left(I_{m}\left(\mathscr{B} / T^{-1} \mathscr{B}\right)+\log g\right) & =m\left(\log g / g_{m}\right) \leqslant m\left(\left(g / g_{m}\right)-1\right) \\
& =\int \sum_{y \in T^{-1} T x} g_{m}(y)\left(\frac{g(y)}{g_{m}(y)}-1\right) d m(x)=0 .
\end{aligned}
$$

Therefore (3) holds.

Now assume (3) holds and we verify (1). By the above reasoning, if $\sigma$ satisfies (3) then $\sigma\left(g / g_{0}-1\right)=\sigma\left(\log g / g_{0}\right)$ so that $g_{\sigma}=g$ a.e. (o). If $f \in$ $C(\bar{X})$ then

$$
\sigma(\overline{\mathcal{E}} f)=\int \mathcal{L} f d \sigma=\int \mathcal{L} f \cdot T d \sigma=\int \sum_{y \in T^{-1} T x} g(y) d \sigma(x)=\sigma(f) .
$$

Notice that we have shown that if $\sigma$ satisfies (3) then

$$
\sigma\left(I_{\sigma}\left(\mathscr{B} / T^{-1} \mathscr{B}\right)+\log g\right)=0 \text {. }
$$

If $|\sigma(\log g)|<\infty$ then this becomes $H_{\sigma}\left(\mathscr{B} / T^{-1 \mathscr{B}}\right)+\sigma(\log g)=0$. If $X_{0}=$ $X=\bar{X}$ and $T$ is expansive then any partition of small diameter is a one-sided generator for every invariant measure and so we can restate (3) as

$$
h_{o}(T)+\sigma(\log g)>h_{m}(T)+m(\log g) \quad \forall m \in M(X, T) .
$$

We shall call a measure $\sigma$ a $g$-measure if $\sigma(X)=1$ and $\sigma$ satisfies (1), (2) and (3). By Theorem 6 and Lemma 9 we know that when $g$ satisfies condition (iii) ${ }_{G}$ there is a unique $g$-measure and it is the measure $\mu$ with the property $\overline{\mathrm{L}}_{\log 8} f \Rightarrow \mu(f)$.

Corollary 11. Let $T: X_{0} \rightarrow X$ satisfy I and let $g \in G\left(X_{0}\right)$ satisfy (ii) $)_{G}$. Let - $\in M(X, T)$ be a $g$-measure. Then $E_{\log g} f(x)=\sum_{y \in T^{-1} x} g(y) f(y)$ defines a positive linear operator on $L^{1}(\sigma)$ and 


$$
\int\left(\varrho_{\log _{g}} f\right) \cdot l d \sigma=\int f \cdot(l \circ T) d \sigma \quad \forall f \in L^{\prime}(\sigma) \forall l \in L^{\infty}(\sigma) \text {. }
$$

ProOF.

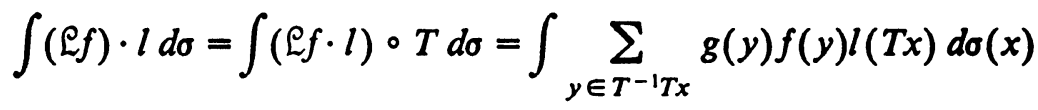

$$
\begin{aligned}
& =\int \sum_{y \in T^{-1} T x} g(y) f(y) l(T y) d \sigma(x)=\int \mathcal{E}(f \cdot(l \circ T)) d \sigma \\
& =\int f \cdot(l \circ T) d \sigma .
\end{aligned}
$$

We now collect together some facts that follow from Theorems 8 and 10 .

Corollary 12. Let $T: X_{0} \rightarrow X$ satisfy I and II and let $\varphi \in C\left(X_{0}\right)$ satisfy (i) and (iii). Let $\lambda, \nu, h, \mu, g$ be the objects determined by Theorem 8 . Then the following are true.

(1) $\mu$ is the unique $g$-measure (recall $g=e^{\varphi} h /(\lambda h \circ T)$ ).

(2) $\mu$ is positive on nonempty open sets and has no atoms.

(3) $\nu \circ T^{-n} \rightarrow \mu$ in $M(\bar{X})$.

(4) If $\psi \in C\left(X_{0}\right)$ also satisfies (i) and (iii) then $\mu_{\varphi}=\mu_{\psi}$ iff $\exists c \in R$ and $f \in C(\bar{X})$ with $\varphi(x)-\psi(x)=f(T x)-f(x)+C, x \in X_{0}$.

(5) $\mu\left(I_{\mu}\left(\mathscr{B} / T^{-1} \mathscr{B}\right)+\varphi\right)>m\left(I_{m}\left(\mathscr{B} / T^{-1} \mathscr{B}\right)+\varphi\right) \forall m \in M(X, T)$ and the value of the left-hand side is $\log \lambda$.

(6) $\lambda$ is also given by

$$
\log \lambda=\lim _{n \rightarrow \infty} \frac{1}{n} \log \bar{\complement}_{\varphi}^{n} 1 .
$$

( $\log \lambda$ is called the pressure of $\varphi$ and denoted $P(\varphi)$.)

(7) $(T, \mu)$ is an exact endomorphism (i.e. $\cap_{n=0}^{\infty} T^{-n} \mathscr{G}_{B}$ consists only of sets of measure 0 or 1 for $\mu$ ).

Proof. (1) This follows by Theorem 6 and Lemma 9.

(2) Let $\varepsilon>0$. By Lemma $5 \exists N>0$ and $b>0$ such that if $x, w \in X$ $\exists y \in T^{-N} x \cap B_{\varepsilon}(w)$ with $\prod_{i=0}^{N-1} g\left(T^{j} y\right)>b$. Therefore

$$
\begin{aligned}
\mu\left(B_{e}(w)\right) & =\int \mathcal{L}_{\log g}^{N} \chi_{B_{e}(w)}(x) d \mu(x) \\
& =\int \sum_{y \in T^{-N_{x} \cap B_{e}(w)}} g(y) \cdots g\left(T^{N-1} y\right) d \mu(x)>b .
\end{aligned}
$$

Hence every $\varepsilon$-ball has $\mu$-measure at least $b$, and so $\mu$ is positive on nonempty open sets.

If $\mu$ has atoms let $x_{0}$ be a point with largest measure among all atoms. Then 


$$
\mu\left(x_{0}\right)=\int \sum_{y \in T^{-1} x} g(y) \chi_{x_{0}}(y) d \mu(x)=g\left(x_{0}\right) \mu\left(T x_{0}\right) .
$$

But $g\left(x_{0}\right)<1$ so by choice of $x_{0}$ we must have $g\left(x_{0}\right)=1$ which contradicts $g>0$ and $\Sigma_{z \in T^{-1} T x_{0}} g(z)=1$.

(3) Let $f \in C(\bar{X})$.

$$
\begin{aligned}
\int f d \nu \circ T^{-n} & =\int f \circ T^{n} d \nu=\frac{1}{\lambda^{n}} \int \overline{\mathscr{L}}_{\varphi}^{n}\left(f \circ T^{n}\right) d \nu \\
& =\frac{1}{\lambda^{n}} \int f \circ \bar{L}_{\varphi}^{n} 1 d \nu \rightarrow \int f \cdot h d \nu \quad \text { by Theorem } 8 .
\end{aligned}
$$

(4) If $\mu_{\varphi}=\mu_{\psi}$ then $g_{\varphi}=g_{\psi}$ a.e. $\left(\mu_{\varphi}\right)$ by part (2) of Theorem 10. Therefore

$$
\varphi-\psi=\log \lambda_{\varphi}-\log \lambda_{\psi}+\log \frac{h_{\varphi}}{h_{\psi}} \circ T-\log \frac{h_{\varphi}}{h_{\psi}} \quad \text { a.e. }\left(\mu_{\varphi}\right) \text {. }
$$

Both sides are continuous on $X_{0}$ and $\mu$ is positive on nonempty open sets by (2) so we get equality on $X_{0}$. Conversely, suppose $\varphi-\psi=f \circ T-f+c$ on $X_{0}$ for some $c \in R$ and some $f \in C(\bar{X})$. Then $p_{\psi}^{n} l=e^{-n c p_{\varphi}^{n}}\left(l e^{f}\right) e^{-f}$ so that

$$
\frac{\mathcal{L}_{\psi}^{n} l}{e^{n c} \lambda_{\varphi}^{n}}=e^{-f} \frac{\mathcal{L}_{\varphi}^{n}\left(l e^{f}\right)}{\lambda_{\varphi}^{n}} \Rightarrow h_{\varphi} e^{-f_{\mu_{\varphi}}}\left(\frac{l e^{f}}{h_{\varphi}}\right) \quad \forall l \in C(\bar{X}) .
$$

Therefore $\lambda_{\psi}=e^{c} \lambda_{\varphi}, h_{\psi}=h_{\varphi} e^{-f}$ and $\mu_{\psi}\left(l / h_{\psi}\right)=\mu_{\varphi}\left(l e^{f} / h_{\varphi}\right)$. The last two equations imply $\mu_{\psi}=\mu_{\varphi}$.

(5) Since $\mu$ is the unique $g$-measure by (1), Theorem 10 asserts that

$$
\mu\left(I_{\mu}\left(\mathscr{B} / T^{-1 \mathscr{B}}\right)+\log g\right)>m\left(I_{m}\left(\mathscr{B} / T^{-1 \mathscr{B}}\right)+\log g\right)
$$

for all $m \in M(X, T)$. Since $\varphi=\log g+\log \lambda+\log h \circ T-\log h$ this is equivalent to $\mu\left(I_{\mu}\left(\mathscr{B} / T^{-1} \mathscr{B}\right)+\varphi\right)>m\left(I_{m}\left(\mathscr{B} / T^{-1} \mathscr{B}\right)+\varphi\right)$ for all $m \in$ $M(X, T)$. We note after the proof of Theorem 10 that $\mu\left(I_{\mu}\left(\mathscr{B} / T^{-1} \mathscr{B}\right)+\right.$ $\log g)=0$ for a $g$-measure so $\mu\left(I_{\mu}\left(\mathscr{B} / T^{-1 \mathscr{B}}\right)+\varphi\right)=\log \lambda$.

(6) This is immediate from Theorem 8 by putting $f \equiv 1$ and taking logarithms.

(7) Let $\mathcal{L}$ denote $\mathcal{L}_{\log g}$. Then $\mathcal{L} f(x)=\Sigma_{y \in T^{-1} x} g(y) f(y)$ defines an operator on $L^{\prime}(\mu)$. We get that $\int\left|\mathbb{R}^{n} f-\mu(f)\right| d \mu \rightarrow 0 \forall f \in L^{\prime}(\mu)$ because for any $\varepsilon>0$ we can choose $l \in C(\bar{X})$ with $\int|f-l| d \mu<\varepsilon$ and then

$$
\begin{aligned}
\int \mid \mathfrak{L}^{n} f- & \mu(f) \mid d \mu \\
& <\int\left|\mathfrak{L}^{n} f-\mathfrak{L}^{n} l\right| d \mu+\int\left|\mathfrak{L}^{n} l-\mu(l)\right| d \mu+|\mu(l)-\mu(f)| \\
& <\int|f-l| d \mu+|\mu(l)-\mu(f)|+\int\left|\mathfrak{L}^{n} l-\mu(l)\right| d \mu,
\end{aligned}
$$

which is small by Theorem 6. We show $(T, \mu)$ is exact by showing 
$E\left(f / \cap_{0}^{\infty} T^{-n \mathscr{B}}\right)=\mu(f)$ a.e. $\forall f \in L^{1}(\mu)$.

$$
\begin{aligned}
\int\left|E\left(f / \bigcap_{0}^{\infty} T^{-n} \mathscr{B}\right)-\mu(f)\right| d \mu< & \int\left|E\left(f / \bigcap_{0}^{\infty} T^{-n}\right)-E\left(f / T^{-N} \mathscr{B}\right)\right| d \mu \\
& +\int\left|E\left(f / T^{-N} \mathfrak{B}\right)-\mu(f)\right| d \mu .
\end{aligned}
$$

The first term is small for large $N$ by the Martingale theorem and the second term equals $\int\left|\left(\mathfrak{L}^{n} f\right) T^{N}-\mu(f)\right| d \mu=\int\left|\mathfrak{L}^{N}-\mu(f)\right| d \mu$ which is small for large $N$ by the above comment.

The fact that $(T, \mu)$ is an exact endomorphism implies strong ergodic properties such as having Lebesgue spectrum of infinite multiplicity [17]. This implies $(T, \mu)$ is strong mixing.

It is natural to ask if $(T, \mu)$ has the property that its natural extension is a Bernoulli shift (see [17] or [18] for the definition of natural extension). We shall show this is so if a reasonable partition exists. Such partitions exist for our examples. Some of our examples will have infinite entropy and so will be generalized Bernoulli shifts in the terminology of [14]. The idea of the proof is taken from [3]. See also [15] for similar ideas.

If $\xi=\left\{A_{1}, A_{2}, \ldots\right\}$ is a partition then $\partial \xi$ will denote $\cup_{i} \partial A_{i}$.

Theorem 13. Let $T: X_{0} \rightarrow X$ satisfy I and II and let $\varphi \in C\left(X_{0}\right)$ satisfy (i) and (iii). Suppose there exists a finite or countable measurable partition $\xi=$ $\left\{A_{1}, A_{2}, A_{3}, \ldots\right\}$ of $X_{0}$ with the following properties: (a) $\left.T\right|_{A_{i}}$ is one-to-one for each $i$, (b) $\mu(\partial \xi)=0$, (c) for each $j, T A_{j}$ is $\mu$-a.e. a union of sets from $\xi$. (d) for all choices of the sequence $i_{n}$ the set $\bigcap_{n=0}^{\infty} T^{-n} \bar{A}_{i_{n}}$ contains at most one point, (e) each $A_{i}$ is a subset of some component of $T^{-1} B_{e_{0}}\left(x_{i}\right)$ for some $x_{i}$. Then the natural extension of $(T, \mu)$ is a (generalized) Bernoulli shift.

Proof. We note that it follows from (d) that $\forall \tau>0 \exists$ integers $d, m$ so that if $x, x^{\prime}$ belong to the same element of the partition

$$
\bigcup_{i=0}^{m} T^{-i}\left\{A_{1}, A_{2}, \ldots, A_{d-1}, \bigcup_{j>d} A_{j}\right\}
$$

then $d\left(x, x^{\prime}\right)<\tau$.

For $d>2$ let $\xi_{d}$ denote the partition $\left\{A_{1}, A_{2}, \ldots, A_{d-1}, \cup_{j>d} A_{j}\right\}$. We shall show $\xi_{d}$ is a weak-Bernoulli partition [7] and the result will then follow by applying the result that an increasing sequence of Bernoulli shifts is a (generalized) Bernoulli shift [14]. When $\xi$ is a finite partition then we do not need to apply this last result. Fix $d \geqslant 2$. We have to show for each $\varepsilon>0$ there exists $N$ such that $n \geqslant N$ implies

(*) $\sum_{P \in V_{0}^{s} T^{-i} \xi_{d}} \sum_{Q \in V_{0}^{s} T^{-i} \xi_{d}}\left|\mu\left(P \cap T^{-(n+s)} Q\right)-\mu(P) \mu(Q)\right|<3 \varepsilon$ for all $s$. 
We can suppose we are in the situation where $\mu$ is the unique $g$-measure for $g \in G\left(X_{0}\right)$ satisfying (iii) ${ }_{G}$. Let $\mathcal{L}$ denote $\mathscr{L}_{\text {log } g}$. Let $\varepsilon>0$ be given. Choose $\delta>0$ so that $(1+\delta)^{3}<\max (1+\varepsilon, 1 /(1-\varepsilon))$. By condition (iii) $)_{G}$ choose $\tau>0, \tau<\varepsilon_{0}$, so that $d\left(x, x^{\prime}\right)<\tau, x, x^{\prime} \in X$ implies

$$
(1+\delta)^{-1}<\prod_{i=0}^{n-1} \frac{g\left(T^{\dot{y}}\right)}{g\left(T^{\dot{y}^{\prime}}\right)}<1+\delta
$$

for all $y \in T^{-n} x$ and all $n \geqslant 1$. Using assumption (d) choose $d_{1}>d$ and $m>0$ so that if $x, x^{\prime}$ are in the same element of $\bigvee_{i=0}^{m} T^{-i} \xi_{d}$ then $d\left(x, x^{\prime}\right)<$ $\tau$, and such that the total measure of the atoms $\cap_{j=0}^{m} T^{-j} A_{i j} \in V_{j=0}^{m} T^{-j} \xi$ with some $i_{j}>d_{1}$ is less than $\varepsilon$.

We shall find $N$ so that (*) holds with $d$ replaced by $d_{1}$. It then follows that (*) holds since $d_{1} \geqslant d$. Also we clearly need only show (*) for $s>m$. Let $s>m$.

Let $C_{j}=A_{j} j<d_{1}$ and $C_{d_{1}}=\bigcup_{j>d_{1}} A_{j}$ so that $\xi_{d_{1}}=\left\{C_{1}, C_{2}, \ldots, C_{d_{1}}\right\}$. Those $\bigcap_{i=0}^{s} T^{-i} C_{j_{i}} \in \bigvee_{i=0}^{s} T^{-i} \xi_{d_{1}}$ with $j_{i} \neq d_{1}$ for $s-m<i \leqslant s$ have measure at least $1-\varepsilon$ by choice of $d_{1}$ and $m$. We shall call these good atoms. Let $P$ be such an atom. Then $P=\cup_{q} P_{q}$ where $P_{q} \in V_{0}^{s} T^{-i} \xi$ and if $P_{q}=\bigcap_{0}^{s} T^{-i} A_{j_{i}}$ then $j_{i}<d_{1}$ for $s-m<i<s$. Suppose $P_{q}=\bigcap_{0}^{s} T^{-i} A_{j_{i}}$.

Consider

$$
\mathfrak{L}^{s-m} \chi_{P_{q}}(x)=\sum_{y \in T^{-(s-m)}(x)} g(y) \cdots g\left(T^{s-m-1} y\right) \chi_{P_{q}}(y) .
$$

This is nonzero iff $x \in T^{s-m} P_{q}$. By assumption (c)

$$
T^{s-m} P_{q}=\bigcap_{i=0}^{m} T^{-i} A_{j_{i+s-m}} \text { a.e. }
$$

By assumption (a) the map $T^{s-m}: P_{q} \rightarrow T^{s-m} P_{q}$ is a bijection and so if $x$, $x^{\prime} \in T^{s-m} P_{q}$ and $y=P_{q} \cap T^{-(s-m)} x$ then $P_{q} \cap T^{-(s-m)} x^{\prime}=y^{\prime}$, the element of $T^{-(s-m)} x^{\prime}$ corresponding to $y$ in the sense of condition $\mathrm{I}$. This is because of assumption (e). Therefore if $x, x^{\prime} \in T^{s-m} P_{q}$ we have

$$
(1+\delta)^{-1}<\mathscr{L}^{s-m} \chi_{P_{q}}(x) / \mathscr{L}^{s-m} \chi_{P_{q}}\left(x^{\prime}\right)<(1+\delta) .
$$

Fix $x^{\prime} \in T^{s-m} P$ and let $c=\mathscr{L}^{s-m} \chi_{P_{q}}\left(x^{\prime}\right)$. Then

$$
(1+\delta)^{-1} c \chi_{T^{s-m P_{q}}}(x)<\mathscr{L}^{s-m} \chi_{P_{q}}(x) \leqslant(1+\delta) c \chi_{T^{s-m P_{q}}}(x)
$$

for all $x$ and we can eliminate $c$ by integrating to give

$$
\begin{aligned}
(1+\delta)^{-2} \frac{\mu\left(P_{q}\right)}{\mu\left(T^{s-m} P_{q}\right)} & \chi_{T^{s-m P_{q}}}(x) \leqslant \mathfrak{L}^{s-m} \chi_{P_{q}}(x) \\
& <(1+\delta)^{2} \frac{\mu\left(P_{q}\right)}{\mu\left(T^{s-m} P_{q}\right)} \chi_{T^{s-m} P_{q}}(x) .
\end{aligned}
$$


Since $\mathcal{L}$ is a positive operator we get

$$
\begin{aligned}
(1+\delta)^{-2} \frac{\mu\left(P_{q}\right)}{\mu\left(T^{s-m} P_{q}\right)} \sum^{n+m} \chi_{T^{s-m P_{q}}}(x)<\mathcal{L}^{n+s} \chi_{P_{q}}(x) \\
<(1+\delta)^{2} \frac{\mu\left(P_{q}\right)}{\mu\left(T^{s-m} P_{q}\right)} \sum^{e^{n+m} \chi_{T^{s-m} P_{q}}(x) .}
\end{aligned}
$$

Multiplying this by $\chi_{Q}$ and integrating gives

$$
\begin{aligned}
(1+\delta)^{-2} \frac{\mu\left(P_{q}\right)}{\mu\left(T^{s-m} P_{q}\right)} & \int \varrho^{n+m} \chi_{T^{s-m P_{q}}} \cdot \chi_{Q} d \mu<\mu\left(P_{q} \cap T^{-(n+s)} Q\right) \\
& <(1+\delta)^{2} \frac{\mu\left(P_{q}\right)}{\mu\left(T^{s-m} P_{q}\right)} \int e^{n+m} \chi_{T^{s-m} P_{q}} \cdot \chi_{Q} d \mu .
\end{aligned}
$$

We know that

$$
T^{s-m} P_{q}=\bigcap_{i=0}^{m} T^{-i} A_{j_{i+s-m}}=P_{q}^{\prime} \text { a.e. }
$$

and $j_{i+s-m}<d_{1}$ for all $i<m$. There are at most $d_{1}^{m}$ choices for these sets $P_{q}^{\prime}$ and since they have boundaries of zero-measure by assumption (b) we can apply Corollary 7 to obtain $N(\varepsilon)$ with the property that $n>N(\varepsilon)$ implies

$$
\left|\int \mathcal{L}^{n+m} \chi_{P^{\prime}} \cdot \chi_{Q} d \mu-\mu\left(P_{q}^{\prime}\right) \mu(Q)\right|<\mu(Q) \frac{\mu\left(P_{q}^{\prime}\right) \delta}{1+\delta}
$$

for all $Q \in \mathscr{B}$ and all sets $P_{q}^{\prime}=\cap_{i=0}^{m} T^{-i} A_{k_{i}}$ with $k_{i}<d_{1}$ all $i$. But since $T^{s-m} P_{q}=P_{q}^{\prime}$ a.e. we get the same property with $P_{q}^{\prime}$ replaced by $T^{s-m} P_{q}$, so that if $n>N(\varepsilon)$ then

$$
(1+\delta)^{-3} \mu\left(P_{q}\right) \mu(Q)<\mu\left(P_{q} \cap T^{-(n+s)} Q\right)<(1+\delta)^{3} \mu\left(P_{q}\right) \mu(Q) .
$$

Summing over $q$ gives

$$
(1-\varepsilon) \mu(P) \mu(Q)<\mu\left(P \cap T^{-(n+s)} Q\right)<(1+\varepsilon) \mu(P) \mu(Q)
$$

for all good atoms $P$ of $V_{0}^{s} T^{-i} \xi_{d_{1}}$ and all $Q \in \mathscr{B}$. Therefore

$$
\sum_{P \in V_{0}^{s} T^{-i} \xi_{d_{1}}} \sum_{Q \in V_{0}^{s} T^{-i} \xi_{1}}\left|\mu\left(P \cap T^{-(n+s)} Q\right)-\mu(P) \mu(Q)\right|
$$

can be split into a sum over the good atoms $P$ and the bad atoms $P$, and we see from the above that the sum is less than $3 \varepsilon$ if $n>N(\varepsilon)$.

In some examples, such as shifts and transformations from $f$-expansions, it is clear that a partition satisfying properties (a)-(e) exists.

2. Invariant measures and equilibrium states. We now describe how to use the results of $\$ 1$ in the problems of invariant measures and of equilibrium states. 
2.1 Invariant measures. Suppose $T: X_{0} \rightarrow X$ satisfies I and II. Suppose we are given $\nu \in M(\bar{X})$ such that $\nu\left(X_{0}\right)=1$. Suppose also that $\nu$ is nonsingular and positively nonsingular for $T$ (i.e., $\nu(E)=0$ implies $\nu\left(T^{-1} E\right)=0$ and $\nu(T E)=0)$. The problem is to see if there exists $\mu \in M(X, T)$ which is equivalent to $\nu$ (i.e., $\mu$ and $\nu$ have the same null sets). The theorem below gives a sufficient condition to ensure the existence of such a $\mu$ with a continuous Radon-Nikodým derivative $d \mu / d \nu$.

Since $T$ is a homeomorphism on a neighborhood of each point of $X_{0}$ and since $\nu$ is positively nonsingular for $T$ we can define the local Radon-Nikodým derivative $d \nu T / d \nu$ at each point of $X_{0}$ to give a map $d \nu T / d \nu: X_{0} \rightarrow R$. This is uniquely defined a.e. $(\nu)$ and we assume it can be chosen to be continuous. Put $\varphi=-\log d \nu T / d \nu \in C\left(X_{0}\right)$. We now see what it means for $\varphi$ to satisfy conditions (i) and (iii). Since

$$
\sum_{y \in T^{-1} x} 1 / \frac{d \nu T}{d \nu}(y)=\frac{d \nu T^{-1}}{d \nu}(x)
$$

condition (i) is equivalent to

(i) $)_{M}$

$$
\frac{d \nu T^{-1}}{d \nu}(x) \leqslant K \quad \forall x \in X .
$$

Using simple properties of Radon-Nikodým derivatives we see that (iii) is equivalent to

(iii) $)_{M}$

$$
\sup _{n>1} \sup _{y \in T^{-n_{x}}} \frac{d \nu T^{n}}{d \nu}\left(y^{\prime}\right) / \frac{d \nu T^{n}}{d \nu}(y)
$$

is bounded for $d\left(x, x^{\prime}\right)<\varepsilon_{0}$ and converges to 1 as $d\left(x, x^{\prime}\right) \rightarrow 0$.

The following simple lemma puts the problem in the context of Theorem 8 .

LEMMa 14. For $\varphi=-\log (d \nu T / d v)$ we have $\overline{\mathrm{e}}_{\varphi}^{*} \nu=\nu$.

Proof. Let $x \in X$ and let $T^{-1}\left(B_{2 \varepsilon_{0}} \cap X\right)=\cup_{i=1}^{\infty} A_{i}(x)$ as in condition I. Let $T_{i}$ denote $\left.T\right|_{A_{i}(x)}$. Then $\left(d \nu T_{i}^{-1} / d \nu\right)\left(T_{i} y\right)\left(d \nu T_{i} / d \nu\right)(y)=1$ so if $E \subset B_{2 \varepsilon_{0}}$ $\cap X$ and $f \in C(X)$,

$$
\begin{aligned}
\int_{E} \overline{\mathcal{L}}_{\varphi} f d \nu & =\int_{E_{i}} \sum_{i=1}^{\infty} \frac{d \nu T_{i}^{-1}}{d \nu}(x) f\left(T_{i}^{-1} x\right) d \nu(x)=\int_{E_{i}=1} \sum_{i=1}^{\infty} f\left(T_{i}^{-1} x\right) d \nu\left(T_{i}^{-1} x\right) \\
& =\sum_{i=1}^{\infty} \int_{T_{i}^{-1} E} f d \nu=\int_{T^{-1} E} f d \nu .
\end{aligned}
$$

The result then follows because $\nu$ is concentrated on $X$.

We can now apply Theorem 8, Corollary 12 and Theorem 13 to get the following result.

TheOREM 15. Suppose T: $X_{0} \rightarrow X$ satisfies conditions I and II and let 
$\nu \in M(X)$ be nonsingular and positively nonsingular for $T$. Suppose $d \nu T / d \nu$ satisfies conditions (i) ${ }_{M}$ and (iii) $)_{M}$ above. Then:

(1) There exists $h \in C(\bar{X}), h>0$ such that $\bar{L}_{\varphi}^{n} f \rightrightarrows h \cdot \nu(f) \forall f \in C(\bar{X})$. (If $x \in X$ then

$$
\mathcal{L}^{n} f(x)=\sum_{y \in T^{-n_{x}}} f(y) / \frac{d \nu T^{n}}{d \nu}(y)
$$

(2) The measure $\mu=h \cdot \nu$ is $T$-invariant and $(T, \mu)$ is an exact endomorphism.

(3) $\nu \circ T^{-n} \rightarrow \mu$ in $M(\bar{X})$.

(4) $\mu$ is the unique member of $M(X, T)$ with the property

$$
\mu\left(I_{\mu}\left(\mathscr{B} / T^{-1} \mathscr{B}\right)-\log \frac{d \nu T}{d \nu}\right)>m\left(I_{m}\left(\mathscr{B} / T^{-1} \mathscr{B}\right)-\log \frac{d \nu T}{d \nu}\right)
$$

$\forall m \in M(X, T)$.

The value of the left-hand side is zero.

(5) If there is a partition $\xi$ with the properties listed in Theorem 13 then the natural extension of $(T, \mu)$ is a Bernoulli shift.

Notice that this theorem does not use any of the results giving properties of $\nu$ (such as Lemma 9) since $\nu$ is given at the start of the problem. When $X_{0}=X=\bar{X}$ and $T$ is expansive then any partition into sets of small diameter is a one-sided generator for every invariant measure and so we can replace the inequality in (4) by

$$
h_{\mu}(T)-\mu\left(\log \frac{d \nu T}{d \nu}\right)>h_{m}(T)-m\left(\log \frac{d \nu T}{d \nu}\right) .
$$

2.2 Equilibrium states. Let $T: X_{0} \rightarrow X$ satisfy conditions I and II. Let $\varphi \in C\left(X_{0}\right)$. We shall say that $\mu \in M(X, T)$ is an equilibrium state for $\varphi$ if

$$
\mu\left(I_{\mu}\left(\mathscr{B} / T^{-1} \mathscr{B}\right)+\varphi\right)>m\left(I_{m}\left(\mathscr{B} / T^{-1} \mathscr{B}\right)+\varphi\right) \quad \forall m \in M(X, T) .
$$

When $X_{0}=X=\bar{X}$ and $T$ is expansive then this definition reduces to the usual definition, namely

$$
h_{\mu}(T)+\mu(\varphi)>h_{m}(T)+m(\varphi) \quad \forall m \in M(X, T)
$$

The results of $\$ 1$ give

Theorem 16. Let $T: X_{0} \rightarrow X$ satisfy I and II and let $\varphi \in C\left(X_{0}\right)$ satisfy (i) and (iii). Then:

(1) $\varphi$ has a unique equilibrium state $\mu$.

(2) $\mu_{\phi}$ has no atoms and is positive on nonempty open sets.

(3) $\left(T, \mu_{0}\right)$ is an exact endomorphism.

(4) If there is a partition $\xi$ with the properties listed in Theorem 13, then the 
natural extension of $\left(T, \mu_{\varphi}\right)$ is a Bernoulli shift.

(5) If $\psi \in C\left(X_{0}\right)$ also satisfy (i) and (iii) then $\mu_{\varphi}=\mu_{\psi} \Leftrightarrow \exists c \in R$ and $f \in C(\bar{X})$ with $\varphi(x)-\psi(x)=f(T x)-f(x)+c \forall x \in X_{0}$.

\section{Examples.}

3.1 Expanding maps. Let $X$ be a compact connected manifold and let $T$ : $X \rightarrow X$ be a $C^{1}$ map of $X$ onto itself. $T$ is an expanding map if there are real numbers $c>0, \lambda>1$ and a Riemannian metric on $X$ for which $\left\|D T^{n} v\right\|>$ $c \lambda^{n}\|v\|, n>0$, for all tangent vectors $v$. Here $D T$ denotes the tangent map of $T$ [23]. If a different metric is chosen then the same condition holds with a new choice of the constant $c$, and it is well known that one can choose a metric for which $c=1$. We shall assume the metric is chosen with this property. Hence $\|D T v\| \geqslant \lambda\|r\|$ for all tangent vectors $v$. In the notation of $\$ 1$ we take $X_{0}=X=\bar{X}$ for this example. Let $d$ be the metric on $X$ determined by the Riemannian metric. There exists $\delta_{0}>0$ such that if $d\left(x, x^{\prime}\right)<\delta_{0}$ then $d\left(T x, T x^{\prime}\right)>\lambda d\left(x, x^{\prime}\right)$. An expanding map is a covering map and so condition I holds, each set $T^{-1} B_{2 \varepsilon_{0}}(x)$ being a union of $k$ sets $A_{1}(x), \ldots, A_{k}(x)$ such that $T$ maps $A_{i}(x)$ homeomorphically to $B_{2 \varepsilon_{0}}(x)$.

LEMMA 17. Condition II holds for an expanding map $T: X \rightarrow X$.

Proof. Let $\tilde{T}: X \rightarrow \tilde{X}$ denote the universal cover of $T: X \rightarrow X$. Let $\varepsilon>0$ be given. For $w \in X$ we have $\tilde{T}^{n}\left(B_{\varepsilon}(w)\right) \supset B_{\lambda^{n}}\left(\tilde{T}^{n} w\right), n>0$ [23]. Choose $N$ so that $\lambda^{N} \varepsilon>\operatorname{diam}(X)$. Then $\tilde{T}^{N}\left(B_{\varepsilon}(w)\right)$ covers $X$ for all $w \in \tilde{X}$ and so $T^{N} B_{\varepsilon}(z)=X \forall z \in X$. Therefore condition II holds.

We now know $T: X \rightarrow X$ satisfies our conditions I and II. We first consider the problem of invariant measures. Let $\nu$ be the smooth probability measure on $X$ determined by the Riemannian metric on $X$. We seek a $T$-invariant probability measure which is equivalent to $\nu$. Consider the linear map $D_{x} T$ : $T_{x} X \rightarrow T_{T x} X$. Using the Riemannian metric we can take its determinant and therefore we can define $T^{\prime}(x)=\operatorname{det}\left(D_{x} T\right)$. Then $(d \nu T / d \nu)(x)=\left|T^{\prime}(x)\right|$ by the charge of variables formula, so the function $\varphi$ defined in $\$ 2.1$ is $\varphi(x)=$ $-\log \left|T^{\prime}(x)\right|$. Since $X$ is connected $T^{\prime}(x)$ always has the same sign. If $T$ is $C^{n}$ then $\varphi$ is $C^{n-1}$. Since the cardinality of $\left\{T^{-1} x\right\}$ is bounded, condition (i) $)_{M}$ holds. If $T$ is $C^{2}$ then we now show condition (iii) ${ }_{M}$ holds. It will be more convenient to show condition (iii) holds for $\varphi=-\log \left|T^{\prime}(x)\right|$.

Suppose $d\left(x, x^{\prime}\right)<\varepsilon_{0}$ and $y \in T^{-n} x$. Then

$$
\left|\sum_{i=0}^{n-1} \varphi\left(T^{\dot{y}}\right)-\varphi\left(T^{\dot{y}^{\prime}}\right)\right|<C \sum_{i=0}^{n-1} d\left(T^{\dot{y}}, T^{\dot{y}^{\prime}}\right)
$$

for some constant $C$ since $\varphi=-\log \left|T^{\prime}\right|$ is $C^{1}$. However $d\left(T^{\prime} y, T^{\dot{y}} y^{\prime}\right)<d(x$, $\left.x^{\prime}\right) / \lambda^{n-i}$ so that 


$$
\left|\sum_{i=0}^{n-1} \varphi\left(T^{i} y\right)-\varphi\left(T^{\dot{y}^{\prime}}\right)\right| \leqslant C \frac{d\left(x, x^{\prime}\right)}{\lambda-1} \text { for all } n>1 .
$$

TheOREM 18. Let $T: X \rightarrow X$ be a $C^{2}$ expanding map. Then there is an invariant measure $\mu$ for $T$ which is equivalent to the smooth measure $\nu$. The following properties hold:

$$
\sum_{y \in T^{-n_{x}}} \frac{f(y)}{\left|\left(T^{n}\right)^{\prime}(y)\right|} \rightarrow^{h \cdot \nu}(f) \quad \forall f \in C(X)
$$

and $\mu=h \cdot \nu$ where $h \in C(X)$ and $h>0$.

(2) $\nu \cdot T^{-n} \rightarrow \mu$ in $M(X)$.

(3) $(T, \mu)$ is an exact endomorphism.

(4) The natural extension of $(T, \mu)$ is a Bernoulli shift.

(5) $\mu$ is the only member of $M(X, T)$ such that

$h_{\mu}(T)-\mu\left(\log \left|T^{\prime}(x)\right|\right)>h_{m}(T)-m\left(\log \left|T^{\prime}(x)\right|\right) \quad \forall m \in M(X, T)$.

(6) $h_{\mu}(T)=\mu\left(\log \left|T^{\prime}(x)\right|\right)$.

$$
\nu \in M(X, T) \Leftrightarrow \sum_{y \in T^{-1} x} \frac{1}{\left|T^{\prime}(y)\right|}=1 \quad \forall x \in X .
$$

Proof. The first six parts are given in Theorem 15. The existence of a partition that will imply the Bernoulli property is given later in Corollary 21 . To prove (7) note that

$$
\nu \in M(X, T) \Leftrightarrow h \equiv 1 \Leftrightarrow \sum_{y \in T^{-1} x} \frac{1}{\left|T^{\prime}(y)\right|}=1 .
$$

Except for parts (3), (4) and (5), the above results have been obtained by Krzyżewski [11].

We now consider equilibrium states for expanding maps. Since $\left\{T^{-1} x\right\}$ is of bounded cardinality every $\varphi \in C(X)$ satisfies condition (i). There are many functions satisfying condition (iii). For example, let $\varphi$ be Hölder continuous $\left(\left|\varphi(x)-\varphi\left(x^{\prime}\right)\right|<M d\left(x, x^{\prime}\right)^{\alpha}\right.$ where $\left.0<\alpha<1\right)$. Then

$$
\begin{aligned}
\left|\sum_{i=0}^{n-1}\left(\varphi\left(T^{\prime} y\right)-\varphi\left(T^{i} y^{\prime}\right)\right)\right| & <M \sum_{i=0}^{n-1} d\left(T^{\dot{y}} y, T^{\dot{y}^{\prime}}\right)^{\alpha}<M \sum_{i=0}^{n-1} \frac{d\left(x, x^{\prime}\right)^{\alpha}}{\lambda^{\alpha(n-i)}} \\
& <\frac{M}{\lambda^{\alpha}-1} d\left(x, x^{\prime}\right)^{\alpha} \text { if } d\left(x, x^{\prime}\right)<\varepsilon_{0} \text { and } y \in T^{-n} x .
\end{aligned}
$$

THEOREM 19. Let T: $X \rightarrow X$ be a $C^{1}$ expanding map and let $\varphi: X \rightarrow R$ be Hölder continuous. Then $\varphi$ has a unique equilibrium state $\mu_{\varphi}$. Also:

(1) $\mu_{\varphi}$ has no atoms and is positive on nonempty open sets.

(2) $\left(T, \mu_{\varphi}\right)$ is exact and has a Bernoulli natural extension.

(3) If $\psi: X \rightarrow R$ is also Hölder continuous then $\mu_{\varphi}=\mu_{\psi} \Leftrightarrow \varphi-\psi=f \circ T-$ 
$f+c$ for some $f \in C(X)$ and some $c \in R$.

(4) Suppose the smooth measure $\nu$ is $T$-invariant and $T$ is $C^{2}$. Then $\nu$ is the unique measure with maximal entropy (i.e., the equilibrium state for the function $0) \Leftrightarrow\left|T^{\prime}(x)\right| \in Z \forall x \in X$.

Proof. All except part (4) follow from Theorem 16. Consider the measure $\mu_{0}$ of maximal entropy and let $\lambda_{0}, \nu_{0}, h_{00}, g_{0}$ be the quantities for the function 0. Then

$$
\int \sum_{y \in T^{-1} x} f(y) d \nu_{0}(x)=\lambda_{0} \int f d \nu_{0} \quad \forall f \in C(X),
$$

so that $\lambda_{0}=k$. We easily see that $h_{0} \equiv 1$. Therefore $g_{0}=1 / k$. If $\nu \in M(X$, $T)$ then $\nu$ is the unique equilibrium state for $\varphi(x)=-\log \left|T^{\prime}(x)\right|$, so that $\nu=\mu_{0}$ iff $g_{\varphi}=g_{0}$. By (7) of Theorem $18, g_{\varphi}(y)=1 /\left|T^{\prime}(y)\right|$, so $\nu=\mu_{0}$ iff $\left|T^{\prime}(y)\right|=k \forall y \in X$.

The same result is true if $X_{0}=X=\bar{X}$ and $T: \bar{X} \rightarrow \bar{X}$ satisfies conditions I, II and also there exist $\delta_{0}>0$ and $\lambda>1$ so that $d(x, x)<\delta_{0} \Rightarrow d(T x$, $\left.T x^{\prime}\right)>\lambda d\left(x, x^{\prime}\right)$. Parts (1) and (2) have recently been obtained by Ledrappier [13]. We stated similar results in [24].

We now indicate why an expanding map has a partition which will satisfy the conditions of Theorem 13 for any reasonable measure. The reasoning is contained in Bowen's construction of Markov partitions for Axiom A diffeomorphisms ([4], [5, p. 79]).

LEMMA 20. Let $X$ be a compact metric space with metric $d$ and let $T: X \rightarrow X$ be such that there exists $\delta_{0}>0$ and $\lambda>1$ with $d\left(T x, T x^{\prime}\right)>\lambda d\left(x, x^{\prime}\right)$ if $d\left(x, x^{\prime}\right)<\delta_{0}$. Then

(1) $T$ has the pseudo orbit shadowing property i.e., $\forall \beta>0 \exists \alpha>0$ with the property that if $\left\{x_{i}\right\}_{0}^{\infty}$ is a sequence of points of $X$ with $d\left(T x_{i}, x_{i+1}\right)<\alpha$ for all $i$ then there exists $x \in X$ such that $d\left(x_{i}, T^{i} x\right)<\beta$ for all $i$. The point $x$ is unique if $2 \beta$ is less than the expansive constant of $T$.

(2) For sufficiently small $\beta$ there is a cover $\left\{R_{1}, \ldots, R_{m}\right\}$ of $X$ by closed sets that intersect only in their boundaries, $X \backslash \cup_{i} \partial R_{i}$ is open and dense, $\operatorname{diam}\left(R_{i}\right)$ $<\beta$ all $i, T\left(\cup_{i} \partial R_{i}\right) \subset \cup_{i} \partial R_{i}$ and if $\operatorname{int}\left(R_{i}\right) \cap T \operatorname{int}\left(R_{j}\right) \neq \varnothing$ then $R_{i} \subset T R_{j}$.

Proof. We sketch the proof. The proof of (1) can easily be written along the lines of Proposition 3.6 of [5].

To prove (2) let $4 \beta$ be less than the expansive constant of $T$ and choose $\alpha$, by part (1), to correspond to $\beta$. Choose $\gamma$ so that $\gamma<\beta, \gamma<\alpha / 2$ and $d\left(x, x^{\prime}\right)<\gamma \Rightarrow d\left(T x, T x^{\prime}\right)<\alpha / 2$. Let $C=\left\{x_{1}, \ldots, x_{r}\right\}$ be a $\gamma$-dense subset of $X$. Put $\Sigma(C)=\left\{q \in \Pi_{0}^{\infty} C \mid d\left(T q_{i}, q_{i+1}\right)<\alpha, i>0\right.$, where $\left.q=\left\{q_{i}\right\}_{0}^{\infty}\right\}$. Define $\theta: \Sigma(C) \rightarrow X$ by letting $\theta(q)$ be the unique point given by part (1). $\theta$ is a continuous map of $\Sigma(C)$ onto $X$. Let $P_{i}=\left\{\theta(q) \mid q_{0}=x_{i}\right\} .\left\{P_{1}, \ldots, P_{r}\right\}$ is 
a closed cover of $X$ and $X \backslash \cup_{i} \partial P_{i}$ is open and dense. We then obtain a collection of disjoint open sets $\left\{D_{1}, \ldots, D_{m}\right\}$ by considering all intersections of sets of the form $\operatorname{int}\left(P_{i} \cap P_{j}\right)$ and $\operatorname{int}\left(P_{k} \backslash P_{e}\right)$. Then put $R_{i}=\bar{D}_{i}$. One can show that $\left\{R_{1}, \ldots, R_{m}\right\}$ is the required cover.

Corollary 21. Let $T: X \rightarrow X$ be as in Lemma 20 and let $\xi$ denote the cover $\left\{R_{1}, \ldots, R_{m}\right\}$. If $\mu$ is an ergodic $T$-invariant measure which is positive on nonempty open sets then $\xi$ is a partition a.e. $(\mu)$ and satisfies all the conditions of Theorem 13.

Proof. Let $\partial \xi=\cup_{i} \partial R_{i}$. Then $\partial \xi \subset T^{-1}(\partial \xi)$ so $\mu(\partial \xi)=0$ or 1 by ergodicity. But $X \backslash \partial \xi$ is open and dense so $\mu(\partial \xi)=0$. Therefore (b) of Theorem 13 holds. Clearly (c) of Theorem 13 also holds by (2) of Lemma 20. Conditions (a), (d), (e) hold because of the local expansion property of $T$.

3.2 Transformations from f-expansion. Let $\bar{X}=[0,1]$ and $X=(0,1)$. Let $\left\{a_{n}\right\}_{-\infty}^{\infty}$ be a finite or countable collection of points in $X$ with $a_{n}<a_{n+1}$ for all $n$ and let $I_{i}=\left(a_{i-1}, a_{i}\right)$. Then $\eta=\left\{I_{n}\right\}_{-\infty}^{\infty}$ is a finite or countable collection of open intervals in $(0,1)$. Put $X_{0}=\cup_{n=-\infty}^{\infty} I_{n}$. Let $T: X_{0} \rightarrow X$ be a map such that $\left.T\right|_{I_{i}}$ is a $C^{1}$ diffeomorphism of $I_{i}$ onto $X$ for each $i>1$. Let $\nu$ be Lebesgue measure on $\bar{X}$. This is clearly nonsingular and positively nonsingular for $T$. The problem of existence of a $T$-invariant probability measure equivalent to $\nu$ has been considered by Rényi [16] and several authors have considered the ergodic properties of this measure. See for example [2], [17] and [19]. We shall deduce the following theorem from the results of $\$ 1$. The theorem was given in [2], except for the variational principle, and has the advantage that its conditions are easily checked for important examples. Similar results can be proved by our method with slightly different hypotheses.

THEOREM 22. Let $T$ be as above. Suppose that

(a) $\left.T\right|_{I_{i}}$ is $C^{2}$ for each $i$.

(b) $\exists k \in Z^{+}$with

$$
\inf _{\substack{x \in I_{i} \\ i \in Z}}\left|\left(T^{k}\right)^{\prime}(x)\right|=\lambda>1 \text {. }
$$

(c)

$$
\sup _{\substack{x, y, z \in I_{i} \\ i \in Z}}\left|\frac{T^{\prime \prime}(x)}{T^{\prime}(y) T^{\prime}(z)}\right|=Q<\infty .
$$

Then

(1) There is a $T$-invariant probability measure $\mu=h \cdot \nu$ where $h \in C(10,1)$, $h>0$.

(2) $\nu \circ T^{-n} \rightarrow \mu$ in $M([0,1])$. 
(3) $(T, \mu)$ is an exact endomorphism and its natural extension is a Bernoulli shift.

(4) $\mu$ is the unique member of $M((0,1), T)$ such that

$$
\begin{aligned}
\mu\left(I_{\mu}\left(\mathscr{B} / T^{-1} \mathscr{B}\right)-\log \left|T^{\prime}\right|\right)>m\left(I_{m}\left(\mathscr{B} / T^{-1} \mathscr{B}\right)-\log \left|T^{\prime}\right|\right) \\
\forall m \in M((0,1), T) .
\end{aligned}
$$

The left-hand side has value 0.

Proof. We are not assuming $\left|T^{\prime}(x)\right|>1 \forall x \in X_{0}$ so we shall have to change the metric so that condition $I$ is verified. To do this we first show $\exists c>0$ so that $\left|T^{\prime}(x)\right|>c \forall x \in X_{0}$. If not then for each $n>1 \exists i_{n} \in Z$ and some $y_{i_{n}} \in I_{i_{n}}$ with $\left|T^{\prime}\left(y_{i_{n}}\right)\right|<1 / n$. From condition (c) we get $\sup _{x \in I_{i_{n}}}\left|T^{\prime \prime}(x)\right|$ $<Q / n^{2}$ so by the mean value theorem

$$
\begin{aligned}
\left|T^{\prime}(z)\right| & <\left|T^{\prime}(z)-T^{\prime}\left(y_{i_{n}}\right)\right|+\left|T^{\prime}\left(y_{i_{n}}\right)\right| \\
& <\frac{Q}{n^{2}}\left|z-y_{i_{n}}\right|+\frac{1}{n}<\frac{Q}{n^{2}}+\frac{1}{n} \quad \text { for all } z \in I_{i_{n}} .
\end{aligned}
$$

For large $n$ this gives $\left|T^{\prime}(z)\right|<\frac{1}{2} \forall x \in I_{i_{n}}$ contradicting the fact that $T$ maps $I_{i}$ onto $X$.

Define a new metric on $(0,1)$ by $\rho\left(x, x^{\prime}\right)=\sup _{y \in T^{-n_{x} ; n>0}}\left|y-y^{\prime}\right|$, and extend to a metric on $[0,1]$. Since $\left|T^{\prime}\right| \geqslant c$ and $\left|\left(T^{k}\right)^{\prime}\right|>\lambda>1$ we have $\left|x-x^{\prime}\right|<\rho\left(x, x^{\prime}\right)<\max \left(1, c^{-k}\right)\left|x-x^{\prime}\right|$ so $\rho$ is equivalent to the usual metric. Condition I clearly holds for the metric $\rho$, with $\varepsilon_{0}=1$. As mentioned before we can check conditions II, (i) ${ }_{M},(\text { iii) })_{M}$ in either metric. We now show condition II holds.

Let $\varepsilon>0$ be given. We need to choose $N$ so that for all $x \in X T^{N}\left(B_{e}(x)\right)$ $=X$. (When we write $T^{N}(E)$ it is understood to mean $T^{N}\left(E \cap T^{-N} X\right)$.) We know $T^{k}$ is a $C^{2}$ diffeomorphism of each member of $\eta_{k}=V_{0}^{k-1} T^{-i} \eta$ onto $X$ and it increases distances by at least a multiple $\lambda$ on each such member. Let $U$ be an open interval of length $2 \varepsilon$. Either $U$ contains a member of $\eta_{k}$, in which case $T^{k} U=X$, or $U$ intersects two members of $\eta_{k}$. Suppose these members are the intervals $(a, b)$ and $(b, c)$. Let

$$
T_{-}^{k}(b)=\lim _{x \rightarrow b-} T_{x}^{k} \in\{0,1\} \text { and } T_{+}^{k}(b)=\lim _{x, b+} T^{k}(x) \in\{0,1\} .
$$

If $T_{-}^{k}(b)=T_{+}^{k}(b)$ then $T^{k}(U)$ has length at least $\lambda \varepsilon$ and is an interval of the form $(0, d)$ or $(e, 1)$. Then $T^{2 k}(U)$ has length at least $\lambda^{2} \varepsilon$ and is again of the form $\left(0, d_{1}\right)$ or $\left(e_{1}, 1\right)$. Hence if $M$ is chosen so that $\lambda^{M} \varepsilon>1$ we have $T^{M k} U=X$. On the other hand if $T_{-}^{k}(b) \neq T_{+}^{k}(b)$ then $T^{k}(U)$ is a union of an interval of form $(0, d)$ and one of the form $(e, 1)$ and has total length $2 \lambda \varepsilon$. At least one of these intervals has length at least $\lambda \varepsilon$ and we can argue with it as above to get $T^{M k} U=X$. Therefore condition II holds. 
We now check condition $(\mathrm{i})_{M}$. For $x, x^{\prime} \in X$,

$$
\begin{aligned}
\frac{d \nu T^{-1}}{d \nu}(x) & =\sum_{y \in T^{-1} x} \frac{1}{\left|T^{\prime}(y)\right|} \\
& >-\sum_{y \in T^{-1} x}\left|\frac{1}{T^{\prime}(y)}-\frac{1}{T^{\prime}\left(y^{\prime}\right)}\right|+\sum_{y^{\prime} \in T^{-1} x^{\prime}} \frac{1}{\left|T^{\prime}\left(y^{\prime}\right)\right|} \\
& =\frac{d \nu T^{-1}}{d \nu}\left(x^{\prime}\right)-\sum_{y \in T^{-1} x}\left|\frac{T^{\prime}\left(y^{\prime}\right)-T^{\prime}(y)}{T^{\prime}(y) T^{\prime}\left(y^{\prime}\right)}\right|>\frac{d \nu T^{-1}}{d \nu}\left(x^{\prime}\right)-Q
\end{aligned}
$$

by condition (c). Since this holds for all $x, x^{\prime} \in X$ condition (i) $)_{M}$ holds.

We now verify condition (iii) $)_{M}$, which is equivalent to verifying condition (iii) for $\varphi(y)=-\log \left|T^{\prime}(y)\right|$. Let $x, x^{\prime} \in X, y \in T^{-n} x$ and let $y^{\prime}$ be the corresponding point of $T^{-n} x^{\prime}$. Then

$$
\begin{aligned}
\varphi\left(T^{\dot{y} y}\right)-\varphi\left(T^{\dot{y} y^{\prime}}\right)= & \varphi^{\prime}\left(z_{i}\right)\left(T^{i} y-T \dot{y}^{\prime}\right) \\
& \quad \text { for some } z_{i} \text { between } T^{\prime} y \text { and } T^{\dot{y}^{\prime}} y^{\prime} \\
& =\frac{\varphi^{\prime}\left(z_{i}\right)}{\left(T^{n-i}\right)^{\prime}\left(w_{i}\right)}\left(x-x^{\prime}\right)
\end{aligned}
$$

for some $w_{i}$ between $T^{i} y$ and $T^{i} y^{\prime}$.

But $\varphi^{\prime}(z)=T^{\prime \prime}(z) / T^{\prime}(z)$ so

$$
\begin{aligned}
\mid \varphi\left(T^{\prime} y\right) & -\varphi\left(T^{\prime} y^{\prime}\right)|=| \frac{T^{\prime \prime}\left(z_{i}\right)}{T^{\prime}\left(z_{i}\right)\left(T^{n-i}\right)^{\prime}\left(w_{i}\right)}\left|x-x^{\prime}\right| \\
& <\frac{Q\left|x-x^{\prime}\right|}{\left|T^{\prime}\left(T w_{i}\right) \cdots T^{\prime}\left(T^{n-i-1} w_{i}\right)\right|}<\frac{Q}{\lambda^{[(n-i-1) / k]} c^{k}}\left|x-x^{\prime}\right| .
\end{aligned}
$$

Therefore

$$
\sum_{i=0}^{n-1}\left|\varphi\left(T^{\dot{y}} y\right)-\varphi\left(T^{\dot{y} y^{\prime}}\right)\right|<\frac{Q}{C^{k}(\lambda-1)}\left|x-x^{\prime}\right| \quad \forall y \in T^{-n} x, n>1 .
$$

Condition (iii) is therefore verified.

The result now follows from Theorem 15. The partition $\eta$ satisfies the conditions of Theorem 13, condition (d) of that theorem following easily from the fact that $d\left(T^{k} x, T^{k} x^{\prime}\right)>\lambda d\left(x, x^{\prime}\right)$ if $x, x^{\prime} \in I_{i}$ for some $i$.

The theorem in [19] which proves the natural extension of $(T, \mu)$ is Bernoulli can be deduced from Theorem 15. The conditions $R_{3}$ and (i) of [19] imply our condition (iii) ${ }_{M}$.

The most important special case of Theorem 22 is when $a_{n}=1 /|n|$ for $n<0, a_{n}=1, n>0$ and $T x=1 / x(\bmod 1)$. In condition (b) we can take 
$k=2$ and $\lambda=4$, and in condition (c) $Q=16$ will suffice. Here

$$
h(x)=\frac{1}{1+x} \cdot \frac{1}{\log 2}
$$

as is well known. Since $\left|\mu\left(\log x^{2}\right)\right|<\infty$ we know $H_{\mu}(\eta)<\infty($ see $[17$, p. 32] or Lemma 23 below) and (4) of Theorem 22 gives that $\mu$ is the unique measure with

$$
h_{\mu}(T)+\mu\left(\log x^{2}\right)>m\left(I_{m}\left(\mathscr{B} / T^{-1} \mathscr{B}\right)+\left(\log x^{2}\right)\right) \quad \forall m \in M((0,1), T) .
$$

Also

$$
h_{\mu}(T)=-\mu\left(\log x^{2}\right)=\pi^{2} / 6 \frac{1}{(\log 2)^{2}} .
$$

The problem of equilibrium states can be considered for transformations arising from $f$-expansions. A result with conditions in the spirit of Theorem 22 is the following.

THEOREM 23. Let $T$ be as above and suppose it satisfies (a), (b) of Theorem 22 and also that $\exists c>0$ with $\left|T^{\prime}(x)\right| \geqslant c \forall x \in X_{0}$. Suppose $\varphi \in C\left(X_{0}\right)$ satisfies

(d) $\exists K$ such that $\sum_{y \in T^{-1} x} e^{\varphi(y)}<K$, and

(e) $|\varphi(x)-\varphi(z)|<M_{i}|x-z|^{\alpha} \forall x, z \in I_{i}$ for some $\alpha<1$ and

$$
\sup _{\substack{y \in I_{i} \\ i \in z}} \frac{M_{i}}{\left|T^{\prime}(y)\right|^{\alpha}}<W<\infty .
$$

Then

(1) $\varphi$ has a unique equilibrium state $\mu_{\varphi}$.

(2) $\mu_{\phi}$ has no atoms and is positive on nonempty open sets.

(3) $\left(T, \mu_{\psi}\right)$ is an exact endomorphism.

(4) $\left(T, \mu_{\varphi}\right)$ has a Bernoulli natural extension.

(5) If $\psi \in C\left(X_{0}\right)$ also satisfies (d) and (e) then $\mu_{\varphi}=\mu_{\psi}$ iff $\exists c \in R$ and $f \in C(\bar{X})$ with

$$
\varphi(x)=\psi(x)+f(T x)-f(x)+c \quad \forall x \in X_{0} .
$$

If the number of intervals $I_{i}$ is finite then any Hölder continuous $\varphi: \bar{X} \rightarrow R$ satisfies (d) and (e).

Proof. By the first part of the proof of Theorem 22 we can change the metric to show $T$ satisfies conditions I and II. The result will follow from Theorem 16 when we have shown $\varphi$ satisfies condition (iii) because (d) is the same as condition (i). Let $y \in T^{-n} x$ then 


$$
\begin{aligned}
\mid \varphi\left(T^{i} y\right) & -\varphi\left(T^{i} y^{\prime}\right)\left|<M_{i}\right| T^{i} y-\left.T^{i} y^{\prime}\right|^{\alpha} \\
& <\frac{M_{i}}{\left|\left(T^{n-i}\right)^{\prime}\left(T^{i} y_{2}\right)\right|^{\alpha}}\left|\left(x-x^{\prime}\right)\right|^{\alpha} \text { for some } y_{2} \text { between } y \text { and } y^{\prime} .
\end{aligned}
$$

Therefore

$$
\sum_{i=0}^{n-1}\left|\varphi\left(T^{j} y\right)-\varphi\left(T^{i} y^{\prime}\right)\right|<\sum_{i=0}^{n-1} \frac{W}{\lambda^{[(n-i-1) / k]_{\alpha}} c^{k \alpha}}\left|x-x^{\prime}\right|^{\alpha}<\frac{W\left|x-x^{\prime}\right|^{\alpha}}{c^{k \alpha}\left(\lambda^{\alpha}-1\right)},
$$

and condition (iii) holds.

For the example $T x=1 / x \bmod 1$, the function $\varphi(x)=\log x^{r}(r>2)$ satisfy these conditions.

The result from [17, p. 32] used above can be extended to give the following result, which can be applied to transformations from $f$-expansions and to shifts.

LEMMA 24. Let $T: X_{0} \rightarrow X$ satisfy conditions I and II in the stronger sense that $T^{-1} X$ is a finite or countable union of open sets $A_{1}, A_{2}, \ldots$ with $\left.T\right|_{A_{i}}$ being a homeomorphism of $A_{i}$ onto $X$ not decreasing any distances. Let $\eta=\left\{A_{1}\right.$, $\left.A_{2}, \ldots\right\}$. Let $g \in G\left(X_{0}\right)$ satisfy (iii) ${ }_{G}$. Then the unique $g$-measure satisfies $H_{\mu}(\eta)<\infty$ iff $|\mu(\log g)|<\infty$.

Proof. From (iii) $)_{G}$ we know $\exists D>0$ such that

$$
D^{-1}<\frac{g(y)}{g\left(y^{\prime}\right)}<D \quad \forall y, y^{\prime} \in A_{i} \text {, all } i \text {. }
$$

If $T_{i}=T \mid A_{i}$ then $\mu\left(A_{i}\right)=\int_{X} g\left(T_{i}^{-1} x\right) d \mu(x)$ so

$$
\sum_{i} \mu\left(A_{i}\right) \log \left(\min _{A_{i}} g\right)<\sum_{i} \mu\left(A_{i}\right) \log \mu\left(A_{i}\right)<\sum_{i} \mu\left(A_{i}\right) \log \left(\max _{A_{i}} g\right) .
$$

Also

$$
\sum_{i} \mu\left(A_{i}\right) \log \left(\min _{A_{i}} g\right)<\int \log g d \mu<\sum_{i} \mu\left(A_{i}\right) \log \left(\max _{A_{i}} g\right) .
$$

But

$$
\left|\log \left(\max _{A_{i}} g\right)-\log \left(\min _{A_{i}} g\right)\right|<\log D
$$

so the outside series either both converge or both diverge. Hence $H_{\mu}(\eta)<\infty$ $\Leftrightarrow|\mu(\log g)|<\infty$.

Corollary 25. Let $X_{0} \rightarrow X$ be as in Lemma 23. If $\varphi \in C\left(X_{0}\right)$ satisfies conditions (i) and (iii) then the unique equilibrium state $\mu_{\varphi}$ of $\varphi$ satisfies $H_{\kappa_{s}}(\eta)<\infty$ iff $\left|\mu_{\varphi}(\varphi)\right|<\infty$. 
3.3 Shifts. The methods of $\$ 1$ generalize those used in [24] for the special case of subshifts of finite type. (The condition $\sum_{n=1}^{\infty} \operatorname{var}_{n}(\varphi)<\infty$ used in [24] implies condition (iii) because $C_{\varphi}\left(x, x^{\prime}\right)<\sum_{i=k}^{\infty} \operatorname{var}_{i}(\varphi)$ if $x$ and $x^{\prime}$ agree in their first $k$ coordinates.) These results are basic to the Ergodic Theory of Axiom A diffeomorphism [3], [5]. The results in $\$ 1$ allow us to deal with the case when the alphabet is infinite. Let $\bar{Z}^{+}$denote the one-point compactification of $Z^{+}=\{1,2,3, \ldots\}$ and let $\infty$ denote the new point. A metric on this space is

$$
\rho(n, m)=\left|\frac{1}{n}-\frac{1}{m}\right|, \quad \rho(n, \infty)=\frac{1}{n} .
$$

Let $\bar{\Omega}$ be the one-sided direct product of an infinite number of copies of $\bar{Z}^{+}$, i.e., $\bar{\Omega}=\Pi_{0}^{\infty} \bar{Z}^{+}$. Let $d$ be the metric on $\bar{\Omega}$ defined by

$$
d\left(\left\{x_{n}\right\}_{0}^{\infty},\left\{y_{n}\right\}_{0}^{\infty}\right)=\sum_{n=0}^{\infty} \frac{\rho\left(x_{n}, y_{n}\right)}{2^{n}} .
$$

Let $\sigma: \bar{\Omega} \rightarrow \bar{\Omega}$ be the shift map. Put $\Omega=\bar{\Omega}, \Omega_{0}=\left\{\left\{x_{n}\right\}_{0}^{\infty} \in \bar{\Omega} \mid x_{0} \neq \infty\right\}$ and $T=\left.\sigma\right|_{\Omega_{0}}$. Then $T: \Omega_{0} \rightarrow \Omega$ satisfies conditions I and II. A function $\varphi: \Omega_{0} \rightarrow R$ satisfies condition (i) if $\Sigma_{i \in Z^{+}} e^{\varphi(i x)}<K \forall x \in \Omega$ (where $i x=\left(i, x_{00}, x_{1}, \ldots\right.$ ) if $\left.x=\left(x_{0}, x_{1}, x_{2}, \ldots\right)\right)$, and satisfies condition (iii) if

$$
C\left(x, x^{\prime}\right)=\sup _{n>1} \sup _{\substack{a_{i} \in Z^{+} \\ 1<i<n}}\left(\sum_{i=0}^{n-1} \varphi\left(T^{i}\left(a_{1} \cdots a_{n} x\right)\right)-\varphi\left(T^{i}\left(a_{1} \cdots a_{n} x^{\prime}\right)\right)\right)
$$

is bounded and $C\left(x, x^{\prime}\right) \rightarrow 0$ as $d\left(x, x^{\prime}\right) \rightarrow 0$. Note that condition (i) implies $\lim _{i \rightarrow \infty} \varphi(i x)=-\infty$.

A simple example where these conditions hold is when $\varphi\left(\left\{x_{n}\right\}_{0}^{\infty}\right)=b_{x_{0}}$ where $\left\{b_{i}\right\}_{0}^{\infty}$ is a given sequence of numbers with $\Sigma_{i \in z^{+}} e^{b_{1}}<\infty$. Then the unique equilibrium state $\mu$ implied by Theorem 16 is the direct product of the measure on $Z^{+}$which gives mass $e^{b_{1}} / \Sigma_{j} e^{b_{j}}$ to $i$. If $\eta$ denotes the natural partition of $X_{0}$ defined by the 0th coordinate then Lemma 23 implies that $H_{\mu}(\eta)<\infty$ iff $|\mu(\varphi)|<\infty$. Hence if $\left|\Sigma_{i \in Z}+b_{i} e^{b_{1}}\right|<\infty$ then $\eta$ is a generator with finite entropy with respect to $\mu$ and we can assert that $\mu$ is the unique measure such that

$$
h_{\mu}(T)+\mu(\varphi)>m\left(I_{m}\left(\mathscr{B} / T^{-1} \mathfrak{B}\right)+\varphi\right) \quad \forall m \in M(\Omega, T) .
$$

We can use the above simple case to give a proof of the existence of a continuous function without a unique equilibrium state on the space $X=$ $\Pi_{0}^{\infty}\{0,1\}$, the one-sided infinite direct product of the two point space $\{0,1\}$. Let $A_{k}=\left\{\left\{x_{n}\right\}_{0}^{\infty} \in X \mid x_{n}=0\right.$ for $\left.0<n<k-1, x_{k}=1\right\}, k=0,1$, $2, \ldots$, and $A_{\infty}=\{0\}$ where 0 is the point $0=(0,0,0, \ldots) . A_{\infty}, A_{0}, A_{1}$, $A_{2}, \ldots$ is a partition of $X$. Let $\left\{a_{k}\right\}_{0}^{\infty}$ be a sequence of real numbers with the 
following three properties

(1) $a_{k} \rightarrow 0$ as $k \rightarrow \infty$,

(2) $\sum_{k=0}^{\infty} e^{a_{0}+\cdots+a_{k}}=1$,

(3) $\sum_{k=0}^{\infty}(k+1) e^{a_{0}+\cdots+a_{k}}<\infty$.

Define $\varphi \in C(X)$ by $\varphi(x)=a_{k}$ if $x \in A_{k}, k=0,1,2, \ldots$, and $\varphi(0)=0$. Then $\varphi$ has exactly two ergodic equilibrium states. The existence of at least two such measures was demonstrated in Fischer [6] and Hofbauer [9], but we present a different proof using the above result on the shift on a space with an infinite state space.

Let $T: X \rightarrow X$ be the shift transformation. Let $A_{0 k}=\left\{\left\{x_{n}\right\} \in A_{0} \mid x_{0}=1\right.$, $x_{i}=0$ for $\left.1<i<k, x_{k+1}=1\right\}, k \geqslant 0$, and let $A_{0}^{\prime}=A_{0} \backslash \cup_{n=0}^{\infty} T^{-n}(0)$ and $A_{0 k}^{\prime}=A_{0 k} \cap A_{0}$. We can take the induced transformation on $A_{0}^{\prime}$ to get a map $T^{*}: A_{0}^{\prime} \rightarrow A_{0}^{\prime}$ defined by $T^{*}(x)=T^{k(x)}(x)$ where $k(x)$ is the smallest natural number with $T^{k(x)} \in A_{0}^{\prime}$. Hence $k(x)=k$ if $x \in A_{0 k}^{\prime}$. Every $m \in M(X, T)$ with $m(0)=0$ has $m\left(A_{0}\right)=m\left(A_{0}^{\prime}\right)$ and determines $m^{*} \in M\left(A_{0}^{\prime}, T^{*}\right)$ by $m^{*}(B)=m\left(B \cap A_{0}\right) / m\left(A_{0}\right)$. Conversely every $\tau \in M\left(A_{0}^{\prime}, T^{*}\right)$ is of the form $m^{*}$ for a unique $m \in M(X, T)$ with $m(0)=0$ provided $\Sigma_{k=0}^{\infty}(k+1) \tau\left(A_{0 k}^{\prime}\right)$ $<\infty$.

Let $\Omega^{\prime}=\Pi_{0}^{\infty} Z^{+}$. Define $\beta: A_{0}^{\prime} \rightarrow \Omega^{\prime}$ by $\beta(x)=\left(w_{0}, w_{1}, w_{2}, \ldots\right)$ where $T^{* n} x \in A_{0 w_{n}-1} . \beta$ is a homeomorphism and $\beta T^{*}=S \beta$. Define $\varphi^{*} \in C\left(A_{0}^{\prime}\right)$ by $\varphi^{*}(x)=a_{0}+\cdots+a_{k} \equiv s_{k}$ if $x \in A_{0 k}^{\prime}$. Then $\varphi^{*} \circ \beta^{-1}\left(w_{0}, w_{1}, w_{2}, \ldots\right)$ $=s_{w_{0}}$ and so $\varphi^{*} \circ \beta^{-1}$ can be considered as defined on $\Omega_{0}$. Condition (2) ensures that the product measure $\pi$ on $\Omega_{0}$ with weights $e^{s_{k}} / \sum_{i=0}^{\infty} e^{s_{1}}$ is the unique equilibrium state for $\varphi^{*} \circ \beta^{-1}$ on $\Omega_{0}$ and the pressure is $\log \left(\Sigma_{n=0}^{\infty} e^{s_{n}}\right)$. This last expression equals 0 by condition (2). $\pi\left(\Omega^{\prime}\right)=1$. Let $\mu^{*}$ denote the member of $M\left(A_{0}^{\prime}, T^{*}\right)$ obtained by pulling $\pi$ back by $\beta$. Then $h_{\mu^{*}}\left(T^{*}\right)+$ $\mu^{*}\left(\varphi^{*}\right)=0$ and $h_{\tau}\left(T^{*}\right)+\tau\left(\varphi^{*}\right)<0 \forall \tau \in M\left(A_{0}^{\prime}, T^{*}\right)$. Condition (3) ensures that $\mu^{*}$ determines a unique $\mu \in M(X, T)$ with $\mu(0)=0$. If $m \in M(X, T)$ and $m(0)=0$ then by direct calculation $m(\varphi)=m\left(A_{0}\right) m^{*}\left(\varphi^{*}\right)$ and by taking natural extensions and using Abramov's formula we have $h_{m}(T)=$ $m\left(A_{0}\right) h_{m^{*}}\left(T^{*}\right)[1]$. Hence

$$
h_{m}(T)+m(\varphi)=m\left(A_{0}\right)\left[h_{m^{*}}\left(T^{*}\right)+m^{*}\left(\varphi^{*}\right)\right] .
$$

Also since $\sum_{k=0}^{\infty}(k+1) m^{*}\left(A_{0 k}^{\prime}\right)<\infty$ it follows that $H_{m^{*}}(\eta)<\infty$ where $\eta$ is the partition $\left\{A_{00}^{\prime}, A_{01}^{\prime}, A_{02}^{\prime}, \ldots\right\}$ of $A_{0}^{\prime}$ (using the simple result that for a sequence $\left\{\alpha_{k}\right\}$ of positive numbers the condition $\Sigma_{k} k \alpha_{k}<\infty$ implies $\left.-\Sigma_{k} \alpha_{k} \log \alpha_{k}<\infty\right)$. Hence $\eta$ is a one-sided generator with finite entropy for each $m^{*}$ and since $\mu^{*}$ is the unique equilibrium state for $\varphi^{*}$ we have $h_{\mu^{*}}\left(T^{*}\right)+\mu^{*}\left(\varphi^{*}\right)=0$ and for all other $m \in M(X, T)$ with $m(0)=0$ we have $h_{m} \cdot\left(T^{*}\right)+m^{*}\left(\varphi^{*}\right)<0$. Therefore $h_{\mu}(T)+\mu(\varphi)=0$ and $h_{m}(T)+m(\varphi)<0$ if $m \in M(X, T), m(0)=0$, and $m \neq \mu$. $\mu$ is ergodic because $\mu^{*}$ is ergodic. 
The point measure, $\delta_{0}$, concentrated at 0 also satisfies $h_{\delta_{0}}(T)+\delta_{0}(\varphi)=0$. Hence the only measures $m \in M(X, T)$ with $h_{m}(T)+m(\varphi)=0$ are convex combinations of $\mu$ and $\delta_{0}{ }^{1}$

From the results of $\$ 1$ we can deduce the following Perron-Frobenius theorem for positive infinite matrices, which generalizes the usual theorem for finite matrices [8].

THEOREM 26. Let $A=\left(a_{i j}\right)_{i, j=1}^{\infty}$ be so that $a_{i j}>0$ for all $i, j$ and satisfy the following two conditions.

(1) $\exists K>0$ such that $\Sigma_{i} a_{i j}<K$ for all $j$.

(2) $\exists C>0$ such that $\sup _{i}\left(a_{i j} / a_{i j^{\prime}}\right)<C$ for all $j, j^{\prime}$ and $\sup _{i}\left(a_{i j} / a_{i j^{\prime}}\right) \rightarrow 0$ as $\left|(1 / j)-\left(1 / j^{\prime}\right)\right| \rightarrow 0$.

Then there exists $\lambda>0, u_{i}>0, v_{i}>0, i>1$ such that

$$
\sum_{i} v_{i}=1, \quad \sum_{i} u_{i} v_{i}=1, \quad \sum_{i} u_{i} a_{i j}=\lambda u_{j}, \quad \sum_{j} a_{i j} v_{j}=\lambda v_{i}
$$

and for each $i\left(1 / \lambda^{n}\right)\left(A^{n}\right)_{i j} \rightarrow u_{j} v_{i}$ uniformly in $j$.

Proof. Let $\bar{X}=\Pi_{0}^{\infty} \bar{Z}^{+}, X=\left\{\left\{x_{n}\right\} \in \bar{X} \mid x_{0} \neq \infty\right\}$ and $X_{0}=\left\{\left\{x_{n}\right\} \in\right.$ $\left.X \mid x_{0} \neq \infty, x_{1} \neq \infty\right\}$. Define $\varphi \in C\left(X_{0}\right)$ by $\varphi\left(x_{0}, x_{1}, \ldots\right)=\log a_{x_{0} x_{1}} \equiv$ $b_{x_{0} x_{1}}$. Then $\varphi$ satisfies conditions (i) and (iii) for the shift transformation. So $\exists \lambda>0, h \in C(X), \nu \in M(X)$ with $\mathfrak{L}_{\varphi} h=\lambda h, \mathfrak{L}_{\varphi}^{*} \nu=\lambda \nu, \nu(h)=1$ and

$$
\frac{\overline{\mathfrak{Q}}_{\varphi}^{n} f}{\lambda^{n}} \rightarrow h \cdot \nu(f) \quad \forall f \in C(\bar{X}) \text {. }
$$

Putting $f \equiv 1$ in the last statement we get $\mathfrak{L}_{\varphi}^{n} 1(x) / \lambda^{n} \rightarrow h(x)$ for $x \in X$. Hence $h(x)$ only depends on $x_{0}$ so define $u_{i}$ to be $h\left(i x_{1} x_{2} \ldots\right)$ for any choice of $x_{1}, x_{2}, \ldots$ Then $\Sigma_{i} u_{i} a_{i j}=\lambda u_{j}$. The corresponding $g$ function is

$$
g(x)=\frac{e^{\varphi(x)} h(x)}{\lambda h(T x)}=\frac{a_{x_{0} x_{1}} u_{x_{0}}}{\lambda u_{x_{1}}} .
$$

Therefore the unique $g$-measure $\mu$ is the Markov chain with initial probability $\mu_{i}$ and transition probabilities

$$
\mu_{i j}=\frac{\mu_{j}}{\mu_{i}} g\left(i j x_{2} x_{3} \ldots\right)=\frac{\mu_{j}}{\mu_{i}} \frac{a_{i j} u_{j}}{\lambda u_{j}} .
$$

Let $v_{i}=\mu_{i} / u_{i}$. Then since $\Sigma_{j} \mu_{i j}=1$ we have $\Sigma_{j} a_{i j} v_{j}=\lambda v_{i}$. Also $\Sigma_{i} u_{i} v_{i}=\Sigma_{i} \mu_{i}$ $=1$. Moreover

$$
\frac{1}{\lambda^{n}}\left(A^{n}\right)_{i j}=\frac{1}{\lambda^{n}} \mathfrak{L}_{\varphi}^{n} f_{i}\left(j x_{1} x_{2} \ldots\right)
$$

where

'F. Ledrappier has also obtained this result by a similar method. 


$$
f_{i}\left(x_{0}, x_{1}, x_{2}, \ldots\right)= \begin{cases}0 & \text { if } x_{0} \neq i \\ 1 & \text { if } x_{0}=i\end{cases}
$$

so for each $i$,

$$
\frac{1}{\lambda^{n}}\left(A^{n}\right)_{i j} \rightarrow h\left(j x_{1} x_{2} \ldots\right) \nu\left(f_{i}\right)=u_{j} v_{i}
$$

uniformly in $j$.

\section{REFERENCES}

1. L. M. Abramov, The entropy of a derived automorphism, Amer. Math. Soc. Transl. (2) 49 (1965), 162-166. MR 22 \#4815.

2. R. L. Adler, F-expansions reoisited, Recent Advances in Topological Dynamics, Lecture Notes in Math., vol. 318, Springer, Berlin, 1973, pp. 1-5. MR 52 \# 10655.

3. R. E. Bowen, Bernoulli equilibrium states for Axiom A diffeomorphisms, Math. Systems Theory 8 (1974/75), 289-294. MR 52 \#8379.

4. - Markoo partitions for Axiom A diffeomorphisms, Amer. J. Math. 92 (1970), 725-747. MR 43 \#2740.

5. Equilibrium states and the engodic theory of Anosou diffeomorphisms, Lecture Notes in Math., vol. 470, Springer, Berlin, 1975.

6. M. E. Fischer, Physica 3 (1967).

7. N. Friedman and D. S. Ornstein, On isomorphism of weak Bernoulli transformations, Advances in Math. 5 (1970), 365-394. MR 43 \#478c.

8. F. Gantmacher, Theory of matrices, Vol. II, Chelsea, New York, 1959. MR 21 \#6372c.

9. F. Hofbauer, Examples for the non-uniqueness of the equilibrium states, Vienna, 1975 (preprint).

10. M. Keane, Strongly mixing 8-measures, Invent. Math. 16 (1972), 309-324. MR 46 \#9295.

11. K. Krzyiewski, On expanding mappings, Bull. Acad. Polon. Sci. Sér. Sci. Math. Astronom. Phys. 19 (1971), 23-24. MR 44 \#6941.

12. F. Ledrappier, Principe veriationel et systemes symboliques, Comm. Math. Phys. 33 (1973), 119-128.

13. ___ Mécanique statistique de l'équilibre pour un revêtement (to appear).

14. D. S. Ornstein, Two Bernoulli shifts with infinite entropy are isomorphic, Advances in Math. 5 (1970), 339-348. MR 43 \#478a.

15. M. Ratner, Anosoo flows with Gibbs measures are also Bernoullian, Israel J. Math. 17 (1974), 380-391. MR 51 \# 10587.

16. A. Rényi, Representations for real mumbers and their ergodic properties, Acta Math. Akad. Sci. Hungar. 8 (1957), 477-493. MR 20 \#3843.

17. V. A. Rohlin, Exact endomorphisms of a Lebesgue space, Amer. Math. Soc. Transl. (2) 39 (1964), 1-36. MR 26 \# 1423.

18. Lectures on the entropy theory of measure-preserving transformations, Russian Math. Surveys 22 (1967), no. 5, 1-52. MR 36 \#349.

19. S. M. Rudolpher and K. M. Wilkinson, A number-theoretic class of weak Bernoulli transformations, Math. Systems Theory 7 (1973), 14-24. MR 48 \#2100.

20. D. Ruelle, Statistical mechanics of a one-dimensional lattice gas, Comm. Math. Phys. 9 (1968), 267-278. MR 38 \#3013.

21. R. Sacksteder, On the convergence to invariant measures (to appear).

22. The measures inoariant under an expanding map (to appear).

23. M. Shub, Endomorphisms of compact differentiable manifolds, Amer. J. Math. 91 (1969), 175-199. MR 39 \#2169.

24. P. Walters, Ruelle's operator theorem and g-measures, Trans. Amer. Math. Soc. 214 (1975), 375-387. 
25. A variational principle for the pressure of continuous transformations, Amer. J. Math. 97 (1975), 937-971. MR 52 \# 11006.

26. ___ Engodic theory-Introductory lectures, Lecture Notes in Math., vol. 458, SpringerVerlag, New York, 1975.

27. M. Waterman, Some ergodic properties of multi-dimensional f-expansions, $Z$. Wahrscheinlichkeitstheorie und Verw. Gebiete 16 (1970), 77-103. MR 44 \# 173.

Matheanatics Institute, University of Warwick, Coventry, England (Current address)

Institut des Hautes Etudes Scientifiques, BURes-sUr-Yvette, France

University of Caltfornia, Berraley, California 94720 